\title{
Hypoxia-reprogrammed tricarboxylic acid cycle promotes the growth of human breast tumorigenic cells
}

\author{
Ke Tang ${ }^{1} \cdot$ Yuandong $\mathrm{Yu}^{1} \cdot$ Liyan $\mathrm{Zhu}^{1} \cdot$ Pingwei $\mathrm{Xu}^{1} \cdot$ Jie Chen ${ }^{1} \cdot$ Jingwei Ma ${ }^{1} \cdot$ Huafeng Zhang ${ }^{1} \cdot$ Haiqing Fang ${ }^{1} \cdot$

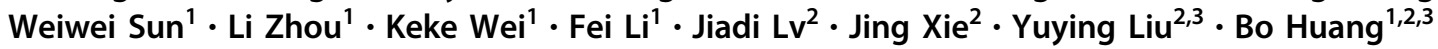

Received: 31 March 2019 / Revised: 11 July 2019 / Accepted: 22 July 2019 / Published online: 13 August 2019

(c) The Author(s) 2019. This article is published with open access

\begin{abstract}
Clinical applications of antiangiogenic agents profoundly affect tumor cell behaviors via the resultant hypoxia. To date, how the hypoxia regulates tumor cells remains unclear. Here, we show that hypoxia promotes the growth of human breast tumorigenic cells that repopulate tumors [tumor-repopulating cells (TRCs)] in vitro and in vivo. This stimulating effect is ascribed to hypoxia-induced reactive oxygen species (ROS) that activates Akt and NF-kB, dependent on the attenuated tricarboxylic acid (TCA) cycle. We find that fumarate is accumulated in the TCA cycle of hypoxic TRCs, leading to glutathione succination, NADPH/NADP ${ }^{+}$decrease, and an increase in ROS levels. Mechanistically, hypoxia-increased HIF$1 \alpha$ transcriptionally downregulates the expression of mitochondrial phosphoenolpyruvate carboxykinase (PCK2), leading to TCA cycle attenuation and fumarate accumulation. These findings reveal that hypoxia-reprogrammed TCA cycle promotes human breast TRCs growth via a HIF-1 $\alpha$-downregulated PCK2 pathway, implying a need for a combination of an antiangiogenic therapy with an antioxidant modulator.
\end{abstract}

\section{Introduction}

Disorganization of tumor vascular networks that generates inter-capillary distances beyond oxygen diffusion range $(100-200 \mu \mathrm{m})$ leads to hypoxia as a common feature in cancers [1]. Moreover, unstable blood flow may further alter tumor oxygen availability [2]. In fact, the median oxygen

These authors contributed equally: Ke Tang, Yuandong Yu, Liyan Zhu

Supplementary information The online version of this article (https:// doi.org/10.1038/s41388-019-0932-1) contains supplementary material, which is available to authorized users.

Bo Huang

tjhuangbo@hotmail.com

1 Department of Biochemistry and Molecular Biology, Tongji Medical College, Huazhong University of Science and Technology, Wuhan 430030, China

2 Department of Immunology, Institute of Basic Medical Sciences \& State Key Laboratory of Medical Molecular Biology, Chinese Academy of Medical Sciences and Peking Union Medical College, Beijing 100005, China

3 Clinical Immunology Center, Chinese Academy of Medical Sciences, Beijing 100005, China partial pressure is $\sim 10 \mathrm{mmHg}$ in most solid tumors, 4-6 folds less than that found in normal tissue [3, 4]. Although it is capable of triggering tumor cell death through apoptosis and/or necrosis both in vitro and in vivo [5-7], hypoxia also promotes tumor angiogenesis, invasiveness, metastasis, and chemo-radioresistance [8,9]. Such paradox might arise from the complex heterogeneity of tumor cells, in which different cellular subpopulations respond to hypoxia differently. This might be particularly true in breast cancer, since multiple breast cancer subtypes coexist within a tumor $[10,11]$. Hypoxia in breast cancer has been widely demonstrated in both mouse and human breast cancers [1214]. Notably, studies have indicated that hypoxia promotes undifferentiated tumorigenic breast cancer cell development and confers them a stem-like phenotype $[15,16]$. In line with this, hypoxia has been shown to promote the upregulation of stemness genes such as Oct3/4, CD133, and Aldhlal and signaling molecules such as NF- $\mathrm{kB}$, Akt, and Wnt are thought to be involved in the process [17, 18]. Notwithstanding such insights into tumor hypoxia, these findings raise a concern on current clinical use of antiangiogenic agents in solid tumor treatment including breast cancer, considering the possibility of that the blood supply blockade might jeopardize the treatment outcome via promoting tumor stemness development. Therefore, more 
mechanistic studies on hypoxia and stemness development are required at least in this type of cancer.

The alteration of glucose metabolism might be of paramount importance for a cell to respond hypoxia. This is because oxygen insufficiency may influence the TCA cycle and glycolysis through hindering the electron transport chain. Notably, this metabolic alteration seems also to have some relationship with the stemness of hypoxic breast cancer cells. For instance, upregulation of glucose metabolic enzymes such as $P H G D H$ has been reported to maintain hypoxic breast cancer stemness via regulating onecarbon metabolism [19, 20]. In addition, upregulation of pyruvate dehydrogenase kinase which regulates the TCA cycle has also been implicated in regulating hypoxic breast cancer stemness [21]. To date, the mechanism through which hypoxia-reprogrammed glucose metabolism confers breast cancer stemness development remains unclear.

Breast stem cell-like cancer cells (SCLCCs) belong to highly tumorigenic subpopulation, which have originally been identified by surface markers $\mathrm{CD} 44^{+} \mathrm{CD} 24^{- \text {llow- }}$ Lineage $^{-}$[22]. However, the use of surface marker to define SCLCCs is controversial due to the unreliability of existing markers [23-25], and that stem and non-stem states are converted bi-directionally [26]. Previously, we applied the biomechanical principles and used soft 3D fibrin gels in $90 \mathrm{~Pa}$ elastic stiffness to culture highly tumorigenic cells, and as few as ten of these cells are able to grow tumors in immunocompetent mice [27]. These mechanically amplified tumorigenic cells are functionally defined as tumorrepopulating cells (TRCs), which are distinct from the SCLCCs that are isolated using conventional cell surface markers, and also appear to be different from the tumorinitiating cells (TICs) which have three distinct subtypes [28]. Moreover, such TRCs can represent SCLCCs in actual tumors such as $\mathrm{CD} 133^{+}$melanoma cells and $\mathrm{ALDH}^{+}$breast cancer cells $[29,30]$. In the present study, we provide evidence that hypoxia promotes the growth of human breast cancer TRCs. The underlying mechanism involves in the downregulation of PCK2 (also known as PEPCK-M) by hypoxia, accumulation of fumarate via reprogrammed TCA cycle and subsequent fumarate-caused increase of ROS levels which promote human breast TRC growth via activating NF-KB and Akt.

\section{Results}

\section{Stem cell-like breast cancer cells are located in hypoxic microenvironments}

Antiangiogenic agents such as sunitinib, when used in cancer patients, generally result in intratumoral hypoxia [18]. To investigate the influence of hypoxia on breast tumor cells, we treated the nude mice, bearing MCF-7 breast cancer with palpable size of $50-100 \mathrm{~mm}^{3}$, with sunitinib $(60 \mathrm{mg} / \mathrm{kg} /$ day $)$. After 35 days, we found that tumors in the sunitinib group were much smaller in size and less vascularized distribution, compared to the control group (Fig. 1a, b). Also, tumors from sunitinib-treated mice displayed multiple areas of intense hypoxia, determined by a hypoxia probe pimonidazole staining (Fig. 1c). Intriguingly, when we used real-time PCR to analyze gene expression in tumor tissues, we found that stemness marker Sox2 was strikingly upregulated along with other stemness markers Oct3/4 and Klf4 in the sunitinib group, compared to the control group (Fig. 1d). Aldehyde dehydrogenase 1 (ALDH1) is commonly used to mark stem cell-like breast cancer cells [17, 30]. Following sunitinib treatment, ALDH1 expression was also upregulated in tumor tissues (Fig. 1e). Surprisingly, more ALDH1 ${ }^{+}$tumor cells were found to be located in hypoxic areas compared to normoxic areas (Fig. 1f). Moreover, such $\mathrm{ALDH}^{+}{ }^{+}$tumorigenic cells in a hypoxic microenvironment showed much higher Ki67 expression (Fig. 1f), indicating a proliferation status. Together, these data suggest that stem cell-like breast cancer cells might prefer to stay in a hypoxic microenvironment.

\section{Hypoxia promotes the growth of human breast tumor-repopulating cells in vitro}

Subsequently, we aimed to validate the above in vivo results in vitro. Despite the importance of stem cell-like tumor cells in tumor initiation, progression, metastasis, and drug resistance, a limitation is that this population belongs to a minor subpopulation and the insufficient quantity restricts extensive mechanistic studies on such stem cell-like cells. To overcome the obstacle, we previously established a mechanics-based 3D soft fibrin gel culture system to select and amplify TRCs [27]. When we seeded ALDH $1^{\text {high }}$ tumor cells from the above breast tumor tissue into the soft 3D fibrin gels, we found that high to $89.7 \%$ (357/400) cells could grow colonies. However, less than $8 \%$ ALDH1 ${ }^{-}$ tumor cells (30/400) grew colonies (Fig. 2a), suggesting ALDH $1{ }^{\text {high }}$ breast tumor cells represent TRCs. Thus, in the following studies, we used human breast cancer TRCs to investigate the mechanistic aspects of how hypoxia promotes tumorigenic breast cell growth. In line with our in vivo data, we found that MCF-7 TRCs grew much better in hypoxia ( $1 \%$ oxygen) than those in normoxia $(21 \%$ oxygen), as evidenced by increased colony sizes, even if colony number had no difference (Fig. $2 b$ and Supplementary Fig. S1). Similarly, this phenomenon was also observed in TRCs from the BT474 and MDA-MB-468 human breast tumor cell line as well as TRCs isolated from three clinical breast tumor samples (Fig. 2c-e). In line with the increased growth, both cell cycle analysis and BrdU 
a
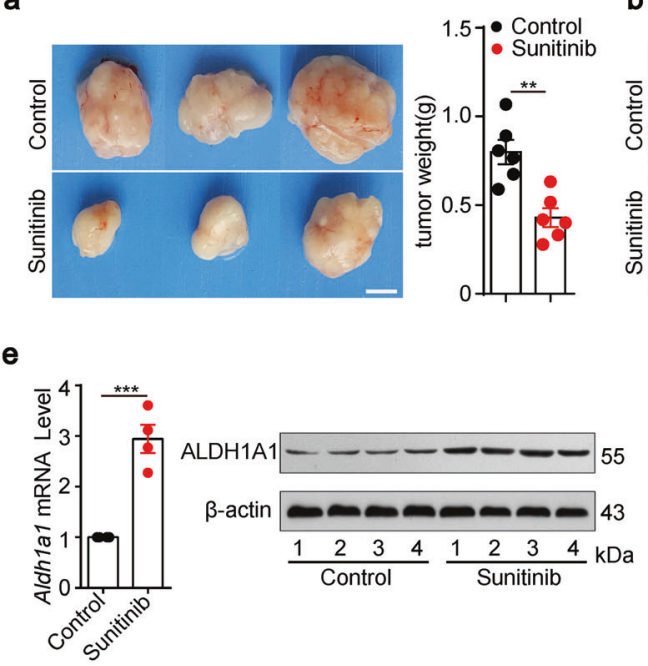

Fig. 1 Hypoxia promotes the growth of stem cell-like breast tumor cells in vivo. $5 \times 10^{6} \mathrm{MCF}-7$ tumor cells were innoculated into the mammary gland of nude mice, followed by intragastric administration of $60 \mathrm{mg} / \mathrm{kg}$ sunitinib per day. a Thirty-five days later, mice were killed and tumor mass was measured (Scale bars, $0.5 \mathrm{~cm}$ ). b Tumor blood vessels were stained with CD31 antibody (green) and the nucleus was stained with DAPI (blue). $\mathbf{c}$ Tumor tissues were stained with pimonidazole adducts (Scale bars, $200 \mu \mathrm{m}$ ). d The expression of

incorporation assay showed that MCF-7 TRCs in hypoxia markedly increased DNA synthesis (S phase), compared to their normoxic counterparts (Fig. 2f). Using a comparable approach, we also observed that MCF-7 TRCs displayed increased growth in $\mathrm{CoCl}_{2}$ treated group which stabilized HIF-1 $\alpha$ protein (Supplementary Fig. S2). Notably, such hypoxia-promoted tumor cell growth seemed to be present in MCF-7 TRCs rather than their differentiated counterparts, because in hypoxic conditions, bulk MCF-7 tumor cells cultured in rigid plate were found to cease growth (Fig. 2g). By contrast, few hypoxic MCF-7 TRCs underwent apoptosis, similar to those TRCs in normoxia (Fig. 2h). Such hypoxic MCF-7 TRCs showed the phenotype of upregulated stemness markers, including Aldhlal, Sox2, CD133, Oct3/4, and Klf4 (Fig. 2i), suggesting that TRCs benefit from hypoxia with a growth and stemness phenotype. To further verify this, we inoculated $1 \times 10^{5}$ hypoxic or normoxic MCF-7 TRCs in NOD-SCID mice, and found that hypoxic TRCs had a greater ability to form a tumor (Fig. 2j). Together, these results suggest that a hypoxic microenvironment is favorable for TRCs proliferation without differentiation.

\section{Hypoxia-promoted TRC growth is mediated by ROS}

Next, we investigated the mechanism through which hypoxia-promoted breast cancer TRCs growth. A key function of oxygen in a cell is to produce $\mathrm{H}_{2} \mathrm{O}$ via c d

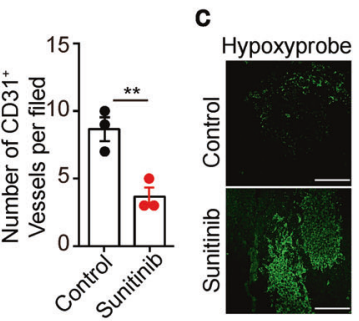

d

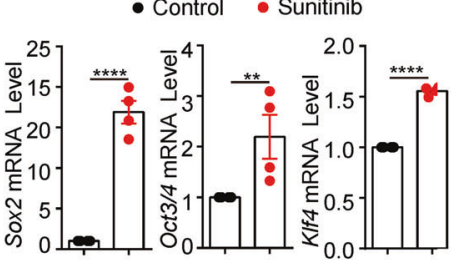

Control Sunitinib

\section{1}

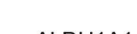

\begin{abstract}
Ki67
\end{abstract}
DAPI
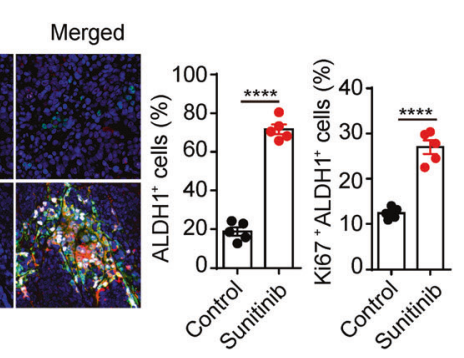

Sox2, Oct3/4, and Klf4 were analyzed by real-time PCR. e ALDH expression in tumor tissues was analyzed by real-time PCR and western blotting, respectively. $\mathbf{f}$ Tumor tissue was stained as described in methods and immunofluorescence staining showed pimonidazole (green), ALDH1 (red), and Ki67 (cyan) merged with DAPI- stained nucleus (blue). Scale bar, $100 \mu \mathrm{m}$. Data shown are representative of three independent experiments and error bars represent mean \pm SEM. $* * p<0.01, * * * p<0.001, * * * * p<0.0001$

mitochondrial oxidative phosphorylation. However, oxygen in the mitochondria can also react with electrons in the absence of proton to generate superoxide, the prototype of reactive oxygen species (ROS). We found that hypoxic MCF-7 TRCs exhibited higher levels of ROS than normoxic TRCs (Fig. 3a). Similar results were also obtained from primary human breast cancer TRCs (Fig. 3b). Intriguingly, blocking ROS levels with antioxidant $N$-acetylcysteine (NAC) effectively hindered TRCs growth in hypoxia but not in normoxia (Fig. 3c, d and Supplementary Fig. S3). By contrast, the addition of hydrogen peroxide to culture medium could result in intracellular ROS elevation (Supplementary Fig. S4) and accompanied with increased MCF-7 TRCs growth in normoxia (Fig. 3e), suggesting that ROS is involved in hypoxia-promoted TRCs growth. In addition, while ROS levels were shown no significant difference between MCF-7 TRCs and bulk tumor cells in normoxia, the bulk ones showed higher levels of ROS than TRCs in hypoxia and the addition of NAC could relieve the hypoxia-induced growth retardation of the bulk tumor cells (Fig. 3f, g), implying that hypoxia-induced ROS production triggers a certain unique pathway that promotes TRCs growth. Although high levels of ROS inevitably cause cellular damage by oxidizing lipids, proteins, and DNA, certain levels of intracellular ROS can actually function as signaling mediators to regulate physiological and biological responses [31]. This led us to hypothesize that TRCs utilize ROS to activate intracellular growth signal(s) so to 

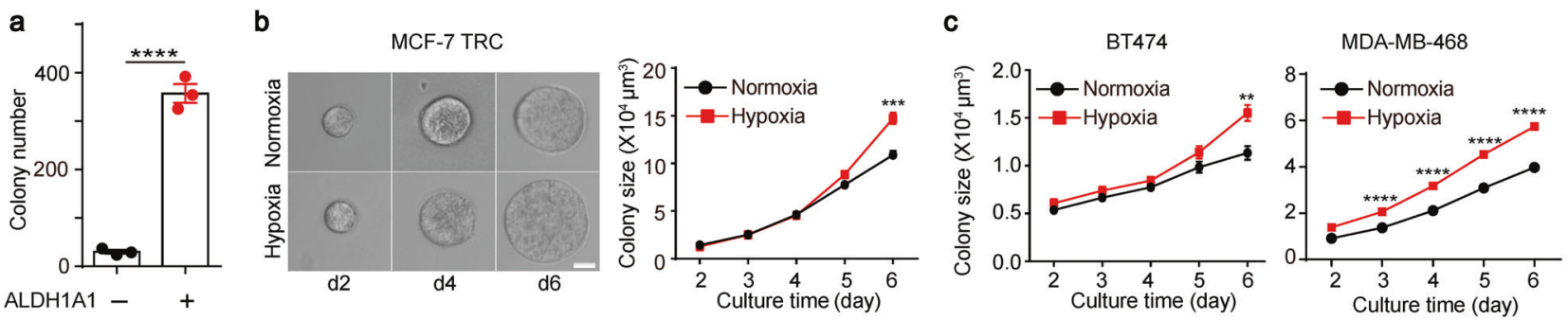

d
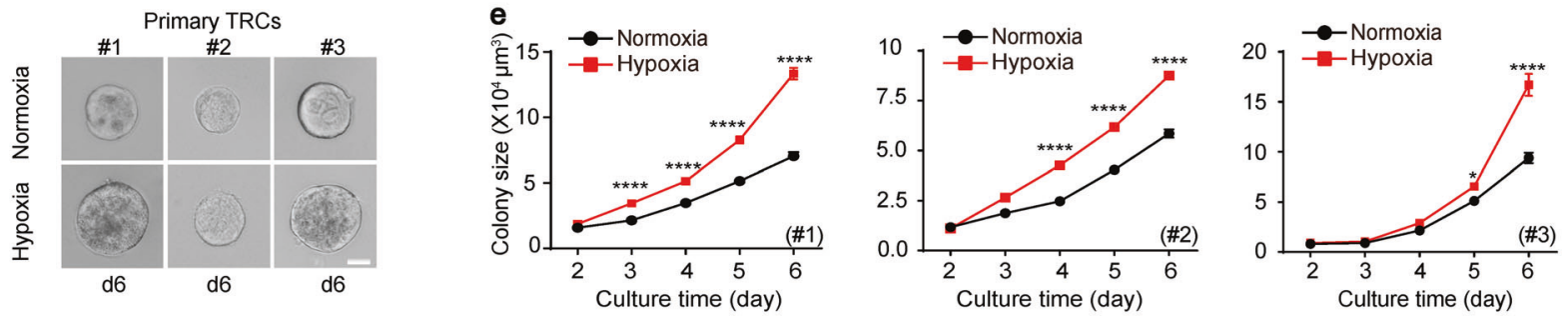
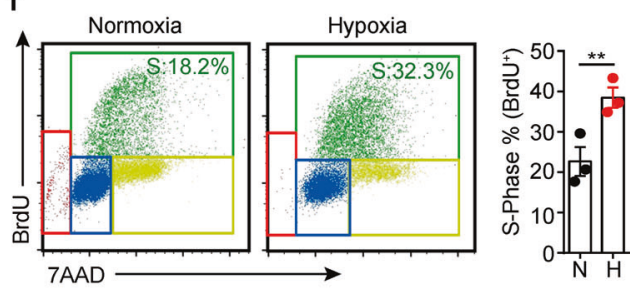

i

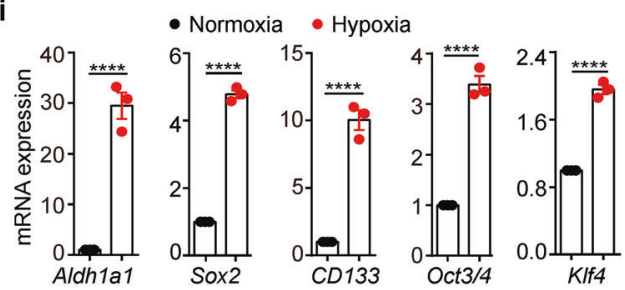

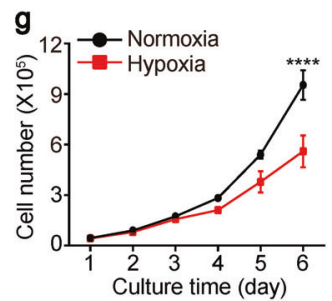

$\mathrm{h}$
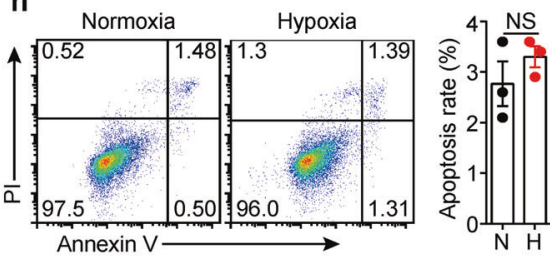

\begin{tabular}{|c|c|c|c|}
\hline Cell type & $\begin{array}{c}\text { Tumor } \\
\text { inoculate }\end{array}$ & $\begin{array}{c}\text { Tumor } \\
\text { incidence }\end{array}$ & $\begin{array}{l}\text { Median } \\
\text { latency }\end{array}$ \\
\hline \multirow{4}{*}{$\begin{array}{l}\text { Hypoxic } \\
\text { MCF-7 TRC }\end{array}$} & {$\left[1 \times 10^{3}\right.$} & 0/12 & $\mathrm{n} / \mathrm{a}$ \\
\hline & $1 \times 10^{4}$ & 0/12 & $n / a$ \\
\hline & $1 \times 10^{5}$ & $7 / 12$ & $15^{* * * * *}$ \\
\hline & $1 \times 10^{3}$ & 0/12 & $\mathrm{n} / \mathrm{a}$ \\
\hline \multirow{2}{*}{$\begin{array}{r}\text { Normoxic } \\
\text { MCF-7 TRC }\end{array}$} & $1 \times 10^{4}$ & 0/12 & $\mathrm{n} / \mathrm{a}$ \\
\hline & {$\left[1 \times 10^{5}\right.$} & $0 / 12$ & $\mathrm{n} / \mathrm{a}$ \\
\hline
\end{tabular}

Fig. 2 Hypoxia promotes the growth of human breast cancer TRCs. a ALDH $1^{\text {high }}$ and ALDH $1^{\text {low }}$ tumor cells were sorted from breast tumor tissue by Aldefluor assay and then were seeded (400 cells per group) into $90 \mathrm{~Pa}$ soft $3 \mathrm{D}$ fibrin gels in $1 \% \mathrm{O}_{2}$ culture incubator. The colony number was calculated. b, c $1.25 \times 10^{3} \mathrm{MCF}-7$ (b), BT474 or MDAMB-468 (c) cells were seeded into 90 Pa soft 3D fibrin gels under $21 \%$ $\mathrm{O}_{2}$ (normoxia) or $1 \% \mathrm{O}_{2}$ (hypoxia) for 6 days, then the colony size was calculated. d, e Primary tumor cells isolated from three human breast cancer samples were seeded into $90 \mathrm{~Pa}$ soft 3D fibrin gels in normoxia or hypoxia for 6 days, the colony size of TRCs was analyzed. $\mathbf{f} 8 \times 10^{3}$ MCF-7 cells were seeded into $90 \mathrm{~Pa}$ soft 3D fibrin gels for 5 days under normoxic or hypoxic conditions, cells were collected and the cell cycle was analyzed by BrdU and 7AAD staining as described in methods. g MCF-7 cells $\left(2 \times 10^{4}\right)$ were cultured in rigid dish under normoxic or hypoxic conditions, the cell number was counted each day. h MCF-7 TRCs were cultured under normoxic or hypoxic condition for 5 days and apoptosis was analyzed by annexin V and PI staining. i Aldh1a1, Sox2, Oct3/4, Klf4, and CD133 mRNA levels in normoxic and hypoxic MCF-7 TRCs were analyzed by real-time PCR. j Hypoxic or normoxic MCF-7 TRCs were subcutaneously inoculated into NOD-SCID mice with different cell counts, and tumor incidence was analyzed. Data shown are representative of three independent experiments and error bars represent mean \pm SEM. ${ }^{*} p<0.05$, $* * p<$ $0.01, * * * p<0.001, * * * * p<0.0001$. NS, no significance. Scale bar, $20 \mu \mathrm{m}$ accelerate their growth in hypoxia. Two key signaling molecules that regulate cellular growth and proliferation, $\mathrm{NF}-\mathrm{kB}$ and Akt, were found to be effectively activated in MCF-7 TRCs and primary human breast cancer TRCs under hypoxic condition, as evidenced by increased phosphorylation of p65 and Akt (Fig. 3h). Notably, $\mathrm{H}_{2} \mathrm{O}_{2}$ has been known to oxidize critical cysteine thiol groups of phosphatases such as PTEN, leading to the phosphorylation of NF- $\mathrm{KB}$ and Akt [32]. In line with this notion, the addition of hydrogen peroxide to cultured MCF-7 TRCs in normoxia led to the increased NF- $\mathrm{kB}$ and Akt phosphorylation (Fig. $3 i)$. However, the addition of NAC could blunt the hypoxiaactivated NF-kB and Akt (Fig. 3j). On the other hand, we blocked the activity of NF- $\mathrm{KB}$ or Akt with inhibitors and found that the promoting effect of hypoxia on TRC growth was prevented but not in normoxic conditions (Fig. 3k-m and Supplementary Fig. S5). Together, these data suggest that hypoxia increases ROS production, allowing ROS as signal molecules to activate NF- $\mathrm{kB}$ and Akt, to ultimately promote human breast cancer TRC growth. 

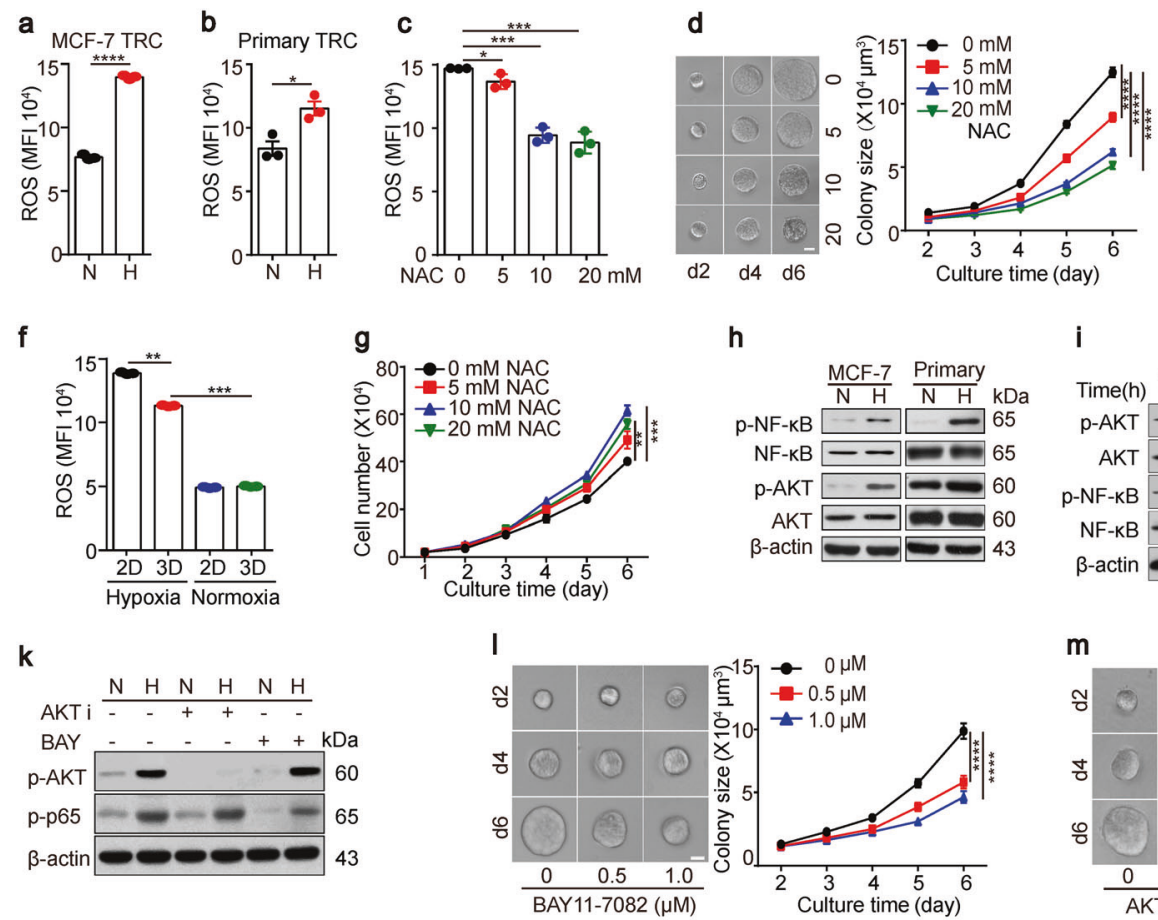
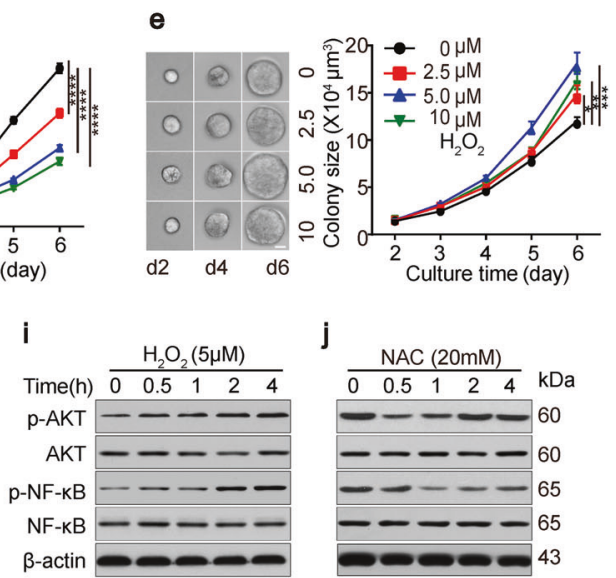

j

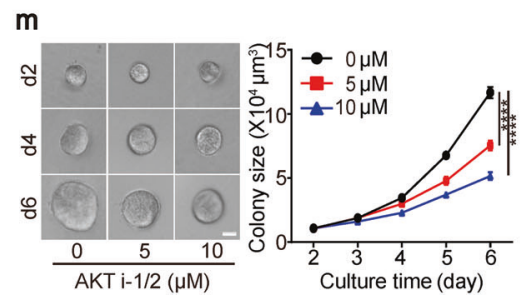

Fig. 3 Hypoxia-promoted TRC growth is mediated by ROS. a, b $8 \times$ $10^{3}$ MCF-7 (a) and primary human breast cancer cells (b) were cultured in $90 \mathrm{~Pa}$ soft 3D fibrin gels in normoxia or hypoxia for 5 days. ROS levels were detected by flow cytometry as described in methods. c, d MCF-7 cells were cultured in $90 \mathrm{~Pa}$ soft 3D fibrin gels under hypoxic conditions in the presence of different doses of NAC. The ROS levels were detected by flow cytometry (c) and the colony size was analyzed (d). e $1.25 \times 10^{3}$ MCF-7 cells were treated with $\mathrm{H}_{2} \mathrm{O}_{2}$ $(2.5 \mu \mathrm{M}, 5.0 \mu \mathrm{M}$, or $10 \mu \mathrm{M})$ in $90 \mathrm{~Pa}$ soft $3 \mathrm{D}$ fibrin gels under normoxic condition and the colony size was analyzed. f MCF-7 cells were cultured in rigid dish (2D) or $90 \mathrm{~Pa}$ soft 3D fibrin gel (3D) under normoxic and hypoxic condition for 5 days, the ROS levels were detected by flow cytometry. g $2 \times 10^{4}$ MCF-7 cells were seeded in rigid dish in the presence of different doses of NAC under hypoxic condition and cell number was counted. h MCF-7 and primary breast

\section{Attenuated TCA cycle is involved in the increased ROS production}

Next, we explored how hypoxia-increased ROS production in breast cancer TRCs. Regarding the TCA cycle coupling with oxidative phosphorylation, carbon flow in the cycle should be affected by oxygen insufficiency. Indeed, an attenuated carbon flow of the TCA cycle in hypoxia was verified by a ${ }^{13} \mathrm{C}$-labeled glucose tracing assay. As shown in (Fig. 4a-e and Supplementary Fig. S6), the relative abundance of the first $(m+2)$ and the second $(m+4)$ TCA cycle intermediates, including citrate, $\alpha-\mathrm{KG}$, fumarate and malate, was much lower in hypoxic TRCs than those in normoxic TRCs. In line with this decreased carbon flow, the expression of citrate synthase (CS), isocitrate dehydrogenase $3 \mathrm{G}$ (IDH3G) and $\alpha$-ketoglutarate dehydrogenase was downregulated in hypoxic MCF-7 TRCs (Fig. 4f), suggesting that the TCA cycle in TRCs is attenuated during hypoxia. To cancer TRCs were cultured in $1 \% \mathrm{O}_{2}(\mathrm{H})$ or $21 \% \mathrm{O}_{2}(\mathrm{~N})$ condition. $24 \mathrm{~h}$ later, $\mathrm{p}-\mathrm{NF}-\mathrm{kB}$ (p65) and p-Akt (ser473) were detected by western blotting. i, j MCF-7 TRCs were cultured under normoxic condition $\left(21 \% \mathrm{O}_{2}\right)$ for 5 days and then treated with $5 \mu \mathrm{M} \mathrm{H}_{2} \mathrm{O}_{2}$ (i), or cultured under hypoxic condition $\left(1 \% \mathrm{O}_{2}\right)$ for 5 days and then treated with $20 \mathrm{mM}$ NAC (j), immunoblotting was carried out for Akt (ser473) and NF- $\kappa B$ analysis. $\mathbf{k}$ MCF-7-TRCs were treated with $10 \mu \mathrm{M}$ AKT $\mathrm{i}-1 / 2$ or $1 \mu \mathrm{M}$ BAY11-7082. Immunoblotting was carried out for Akt

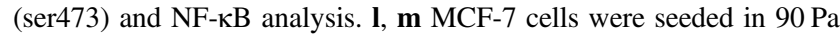
soft 3D fibrin gels and treated with different doses of BAY11-7082 or AKT $\mathrm{i}-1 / 2$ under hypoxic condition $\left(1 \% \mathrm{O}_{2}\right)$ for the indicated days. The colony size was presented photographically and graphically. Data shown are representative of three independent experiments and error bars represent mean \pm SEM. $* p<0.05$, **p $p<0.01$, *** $p<0.001$, $* * * * p<0.0001$. Scale bar, $20 \mu \mathrm{m}$

verify whether this attenuated TCA cycle is associated with the increased ROS levels and TRCs growth, we knocked down malate dehydrogenase (MDH) to restrain carbon flow in the TCA cycle in hypoxia. We found that TRCs growth was stimulated, simultaneous with increased ROS levels (Fig. 4g, h). Consistently, the use of NAC to reduce ROS levels led to decreased TRCs growth (Supplementary Fig. S7), suggesting that the attenuated TCA cycle increases ROS production for hypoxia-induced breast cancer TRCs growth. To further validate this, we additionally knocked down IDH3G to restrain the carbon flow from the upstream of the TCA cycle. Surprisingly, such IDH3G knockdown did not promote but inhibited the hypoxia-induced TRCs growth, concomitant with decreased ROS levels (Fig. 4i, j), and replenishing hydrogen peroxide rescued the impaired TRCs growth (Fig. 4k). These paradoxical results might result from the altered intermediate metabolite(s) of the TCA cycle, considering that enzymatic blockade may result 
a

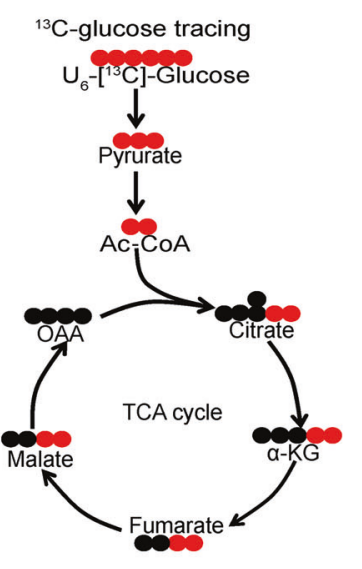

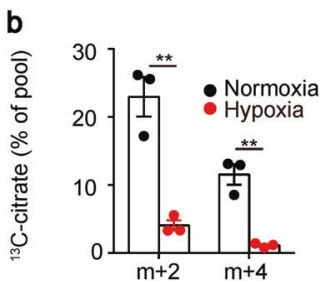

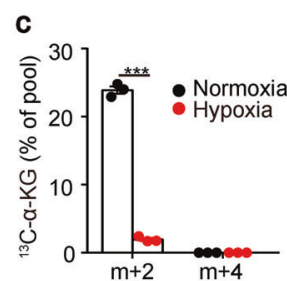

f

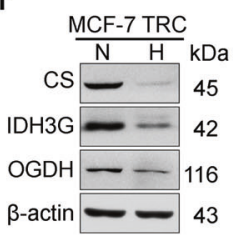

g
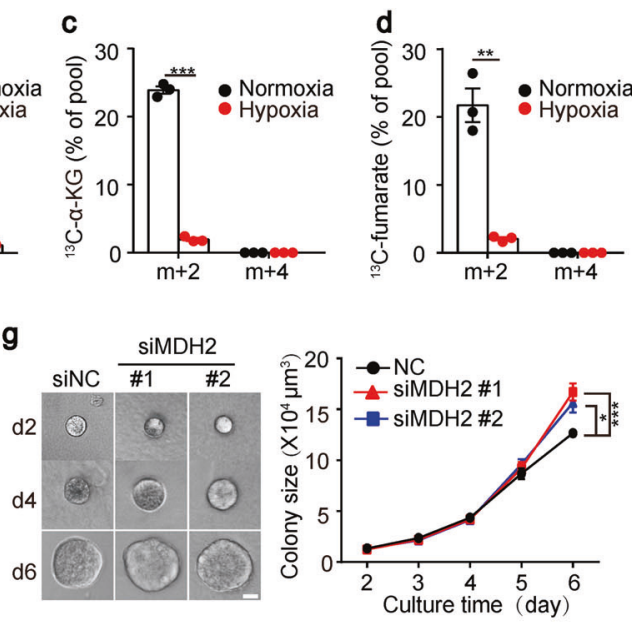

e

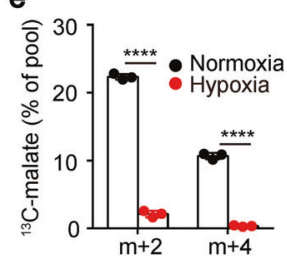

h

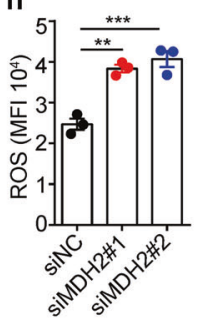

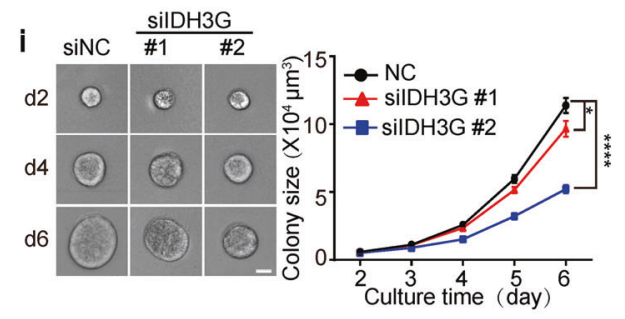

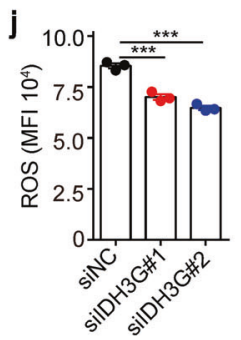

k

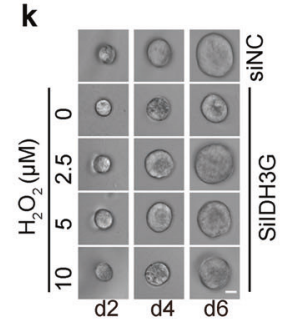

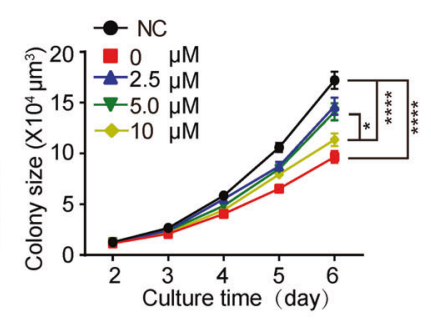

Fig. 4 An attenuated TCA cycle is involved in the increased ROS production. a A metabolic model describing the flow of [U6]- ${ }^{13} \mathrm{C}$ glucose into the TCA cycle. b-e MCF-7 cells were seeded in $90 \mathrm{~Pa}$ soft 3D fibrin gels for 4 days under normoxic or hypoxic conditions, and then incubated with ${ }^{13} \mathrm{C}$-glucose for another $24 \mathrm{~h}$, LC-MS were performed for $\mathrm{m} 0, \mathrm{~m}+2$ or $\mathrm{m}+4$ labeled citrate, $\alpha-\mathrm{KG}$, fumarate and malate detection. $\mathbf{f} \mathrm{CS}, \mathrm{IDH} 3 \mathrm{G}$ and $\mathrm{OGDH}$ expression in hypoxic or normoxic MCF-7 TRCs were analyzed by western blotting. $\mathbf{g}$, h MCF7 cells were transfected with MDH2 siRNA and then cultured in $90 \mathrm{~Pa}$ soft 3D fibrin gels under hypoxic condition $\left(1 \% \mathrm{O}_{2}\right)$. The colony size was analyzed (g) and ROS levels were detected by flow cytometry (h). i, j MCF-7 cells were transfected with IDH3G siRNA and then cultured in 90 pa soft 3D fibrin gels under hypoxic condition $\left(1 \% \mathrm{O}_{2}\right)$. The colony size was analyzed (i) and ROS levels were detected by flow cytometry (j). k MCF-7 cells were transfected with IDH3G siRNA and treated with different concentrations of $\mathrm{H}_{2} \mathrm{O}_{2}$ in $90 \mathrm{~Pa}$ soft $3 \mathrm{D}$ fibrin gels under hypoxic condition $\left(1 \% \mathrm{O}_{2}\right)$. The colony size was presented photographically and graphically. Data shown are representative of three independent experiments and error bars represent mean \pm SEM. ${ }^{*} p<0.05, * * p<0.01, * * * p<0.001, * * * * p<0.0001$. Scale bar, $20 \mu \mathrm{m}$

the PDK1 expression were increased in hypoxic TRCs (Supplementary Fig. S8). To reconcile this paradox, we speculated that hypoxic TRCs might use glutamine as a supplemental carbon source, considering the important role of glutamine in supporting the TCA cycle [34]. By conducting ${ }^{13} \mathrm{C}$ labeled glutamine tracing assay, we found that more glutamine-derived intermediate metabolites $(\mathrm{m}+5 \alpha$ $\mathrm{KG}$ and $\mathrm{m}+4$ succinate, fumarate and malate) were detected in hypoxic TRCs relative to normoxic ones (Fig. $5 \mathrm{~b}, \mathrm{c})$. In addition, we found that a lower glutamine level inhibited hypoxic TRC growth, but did not affect normoxic TRC growth (Supplementary Fig. S9), suggesting that hypoxic breast TRCs mobilize glutamine metabolism to elevate the levels of $\alpha$-ketoglutarate, succinate, fumarate and malate. To verify which metabolite(s) is involved in ROS production in hypoxic TRCs, we used dimethyl 2oxoglutarate (MOG), dimethyl fumarate (DMF), succinic acid dimethyl ester (SAD), the corresponding cell membrane-permeable forms of $\alpha-\mathrm{KG}$, fumarate and the above intermediate metabolites. Here we also found that 

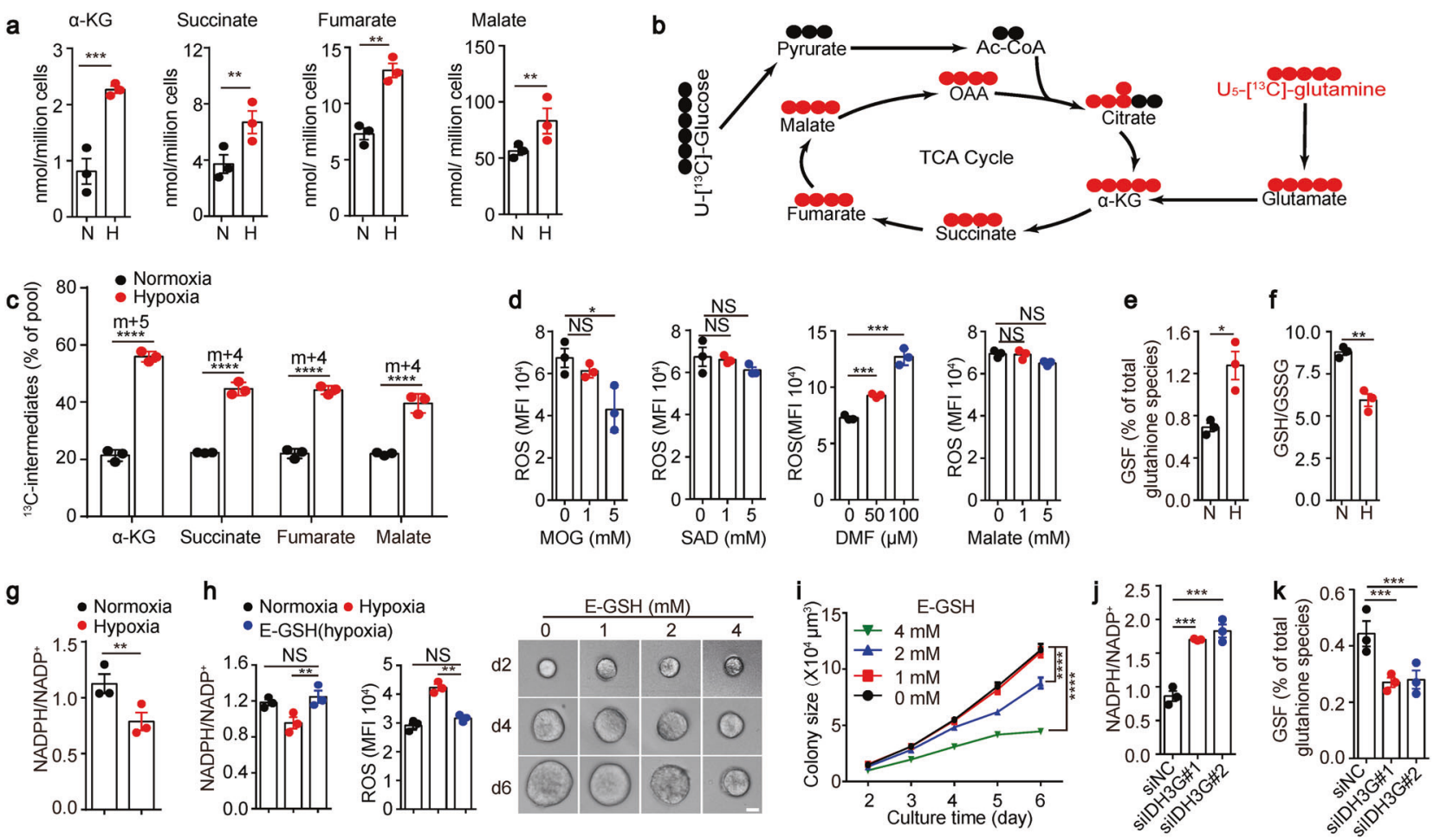

Fig. 5 Fumarate accumulation increases ROS levels via forming succinic glutathione in hypoxic breast cancer TRCs. a Levels of $\alpha$-ketoglutarate, succinate, fumarate and malate in hypoxic or normoxic breast TRCs were analyzed by LC-MS. b A metabolic model describing the flow of [U5] $-{ }^{13} \mathrm{C}$-glutamine into the TCA cycle. $\mathbf{c}$ MCF7 cells were seeded in $90 \mathrm{~Pa}$ soft 3D fibrin gels for 4 days under normoxic or hypoxic conditions, and then incubated with ${ }^{13} \mathrm{C}$ glutamine for another $24 \mathrm{~h}$, LC-MS were performed for $\mathrm{m}+5$ isoform of $\alpha-K G$ and $m+4$ isoforms of succinate, fumarate, and malate. d MCF-7 cells were seeded in $90 \mathrm{~Pa}$ soft 3D fibrin gels and treated with different doses of MOG, SAD, DMF, and malate for 5 days in hypoxia, ROS levels were determined by flow cytometry. e GSF levels

succinate (Supplementary Fig. S10), and L-malate to treat hypoxic TRCs, respectively. Notably, we found that only DMF increased ROS levels (Fig. 5d), consistent with a previous report [35]. In addition, we also tested fumarate monomethyl fumarate (MMF), another cell membranepermeable form of fumarate. We found that the levels of fumarate in the hypoxic TRCs were also elevated, concomitant with increased ROS levels (Supplementary Fig. S11). Fumarate is a carbon-carbon double bond unsaturated electrophilic metabolite that can covalently bind to a thiolate anion of cysteine residues to form a S-(2-succinyl)cysteine (2SC) adduct, known as protein succination [36]. Fumarate covalently binds glutathione, thus producing succinated glutathione (GSF); whereas GSF can act as a substrate of glutathione reductase, thus consuming NADPH and elevating ROS levels via hindering glutathione production [37]. In line with this notion, hypoxic breast cancer TRCs showed much higher GSF than normoxic TRCs (Fig. 5e and Supplementary Fig. S12). Furthermore, the result of normoxic or hypoxic breast TRCs were analyzed by LC-MS as described in methods. f, g GSH/GSSG, NADPH/NADP+ratio from normoxic and hypoxic breast cancer TRCs were analyzed by assay kits as described in methods. $\mathbf{h}, \mathbf{i}$ MCF-7 cells were seeded in soft 3D fibrin gels and treated with different doses of ethyl-GSH for 5 days in hypoxia, NADPH/NADP + ratio, ROS levels (h) and colony size (i) were analyzed. Scale bar, $20 \mu \mathrm{m}$. j, k MCF-7 cells were transfected with IDH3G siRNA and cultured in $90 \mathrm{~Pa}$ soft 3D fibrin gels under hypoxic condition for 5 days, the NADPH/NADP + ratio (j) and GSF (k) were analyzed. Data shown are representative of three independent experiments and error bars represent mean \pm SEM. $* p<0.05$, $* * p<$ $0.01, * * * p<0.001, * * * * p<0.0001$. NS, no significance

from ${ }^{13} \mathrm{C}$-labled glutamine showed that glutamine-derived fumarate resulted in more GSF ( $\mathrm{m}+4$ form) in hypoxic TRCs (Supplementary Fig. S13). Also, the ratios of GSH/ GSSG and NADPH/NADP ${ }^{+}$were lower in hypoxic TRCs (Fig. 5f, g). Moreover, provision of ethyl-GSH (E-GSH), a cell-permeable derivative of GSH, could rescue NADPH levels to the extent of that in normoxic TRCs, decreased ROS levels, and hindered hypoxia-promoted TRCs growth (Fig. 5h, i). In addition, the increase of GSF and decrease of NADPH could be remedied by IDH3G knockdown, but became worse by fumarate hydratase knockdown (Fig. 5j, k and Supplementary Fig. S14). Considering the higher ROS levels in hypoxic differentiated MCF-7 cells, we additionally measured GSF and the GSH/GSSG ratio, which showed that GSF was higher and the GSH/GSSG ratio was reduced in differentiated MCF-7 cells as compared to the MCF-7 TRCs after the induction of hypoxia (Supplementary Fig. S15). Thus, it may stand to reason that too much ROS was generated in the hypoxic differentiated MCF-7 
cells which caused growth retardation in these cells. Together, these data suggest that fumarate accumulation causes the formation of succinic glutathione, leading to the increased, and yet suitable, ROS levels in hypoxic breast cancer TRCs.

\section{PCK2 downregulation causes fumarate accumulation in hypoxic breast cancer TRCs}

Subsequently, we investigated how hypoxia caused fumarate accumulation in breast cancer TRCs. Mitochondrial phosphoenolpyruvate carboxykinase (PCK2) is a key enzyme that regulates the carbon flow of the TCA cycle by the conversion of mitochondrial oxaloacetate (OAA) to phosphoenolpyruvate (PEP). Previously, we found that the downregulation of PCK2 leads to increased fumarate levels in B16 melanoma TRCs [38]. Here, we found that PCK2 was highly expressed in normoxic breast cancer TRCs but much lowly expressed under hypoxic conditions (Fig. 6a). Utilizing a tetracycline-induced PCK2 overexpression system (Supplementary Fig. S16), we found that PCK2 overexpression led to decreasing the levels of fumarate, ROS and GSF, but increasing NADPH/NADP ${ }^{+}$and GSH/GSSG in hypoxic breast cancer TRCs (Fig. 6b-d). As a result, PCK2 overexpression led to a decreased proliferation and stemness gene expression in hypoxic TRCs (Fig. 6e-g). In addition, PCK2 knockdown promoted TRC growth (increased colony size) in normoxia (Supplementary Fig. S17). Given the catalysis of OAA by PCK2, we additionally analyzed OAA, and found that OAA levels were low in normoxic TRCs but were high in hypoxic TRCs; however, OAA levels were decreased as a result of PCK2 overexpression (Fig. 6h), implying that PCK2 downregulation causes OAA accumulation. Thus, we hypothesized that increased OAA levels impeded carbon flow and resulted in fumarate accumulation in hypoxic TRCs. To test this, we used ${ }^{13} \mathrm{C}$-glucose to trace carbon flow in the TCA cycle. As expected, $\mathrm{m}+2$ and $\mathrm{m}+4$ forms of citrate, $\alpha$-ketoglutarate, fumarate and malate were elevated in hypoxic PCK2overexpressing TRCs (Fig. 6i-l and Supplementary Fig. $\mathrm{S} 18)$. In addition, the result from ${ }^{13} \mathrm{C}$-glutamine tracing showed that PEP from glutamine-derived OAA $(\mathrm{m}+3$ form) was decreased in hypoxic TRCs, which could be rescued by PCK2 overexpression (Supplementary Fig. S19). Such PCK2 overexpression decreasing intermediate metabolites and ROS levels (Supplementary Fig. S20 and Fig. $6 \mathrm{~m}, \mathrm{n})$ and hypoxic TRCs $\left(\mathrm{ALDH} 1^{+}\right.$cells) proliferation was observed in nude mice inoculated with PCK2overexpressing TRCs, followed by sunitinib treatment and Ki67 staining (Fig. 6o, p). Consistently, an increased $\mathrm{NADPH} / \mathrm{NADP}^{+}$ratio and a decreased tumor growth were also observed (Fig. 6q, r). In addition, the expression of stemness genes were downregulated in the PCK2- overexpressing group (Fig. 6s). Together, these data suggest that PCK2 downregulation can cause OAA accumulation, thus hindering the carbon flow of the TCA cycle and leading to fumarate accumulation in hypoxic breast cancer TRCs.

\section{HIF-1a negatively regulates PCK2 expression}

Finally, we investigated the mechanism by which PCK2 was downregulated in hypoxic breast cancer TRCs. Upregulation of hypoxia-induced factors (HIF) is a universal response of cells to hypoxia. Previous study had showed that HIF could regulate PCK2 expression in human lung cancer cells [39]. Given that HIFs profoundly regulate glucose metabolism including the TCA cycle, we determined HIF- $\alpha$ protein levels in hypoxic breast cancer TRCs by western blot. Both normoxic and hypoxic breast cancer TRCs highly expressed HIF- $2 \alpha$, however, HIF- $1 \alpha$ was strikingly upregulated only in hypoxic TRCs (Fig. 7a), prompting us to hypothesize that the expression of PCK2 was regulated by HIF-1 $\alpha$. When we used siRNAs to knockdown HIF-1 $\alpha$, we found that PCK2 expression was upregulated in hypoxic breast cancer TRCs (Fig. 7b). Correspondingly, overexpression of HIF-1 $\alpha$ resulted in the PCK2 downregulation (Fig. 7b), suggesting that PCK2 is regulated by HIF- $1 \alpha$. In line with this result, a ${ }^{13} \mathrm{C}$-glucose tracing assay showed that HIF- $1 \alpha$ knockdown promoted carbon flow of the TCA cycle (Fig. 7c-e and Supplementary Fig. 21). Moreover, analysis with the UCSC Genome Browser and JASPAR revealed the presence of multiple consensus cis-elements for HIF- $1 \alpha$ binding on the promoter of PCK2, and a Chip-PCR assay indicated that HIF-1 directly bound to the PCK2 promoter (Fig. 7f, g). We then conducted a PCK2 promoter luciferase assay, showing that HIF- $1 \alpha$ knockdown increased luciferase activity but HIF- $1 \alpha$ overexpression decreased the luciferase activity (Fig. 7h). Since fumarate is able to regulate HIF-1 $\alpha$ stability via inhibiting a-ketoglutarate, we additionally used MMF to treat normoxic breast cancer TRCs. As expected, HIF- $1 \alpha$ expression was enhanced and PCK2 expression was further decreased (Fig. 7i). Meanwhile, the phosphorylation of p65 and Akt were increased by MMF treatment (Supplementary Fig. S22). In line with this, TRC growth (colony size) were accelerated after MMF treatment in normoxia (Fig. 7j). Together, these results suggest that hypoxia-induced HIF- $1 \alpha$ negatively regulates PCK2 expression and fumarate accumulation further strengthens this feedback in breast cancer TRCs.

\section{Discussion}

Hypoxia as a hallmark of cancer is intensively investigated in various tumor types. To date, how hypoxia differentially 


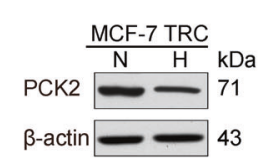

- CON Vec • OE-PCK2
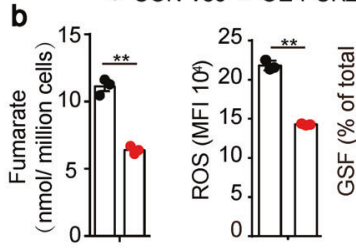
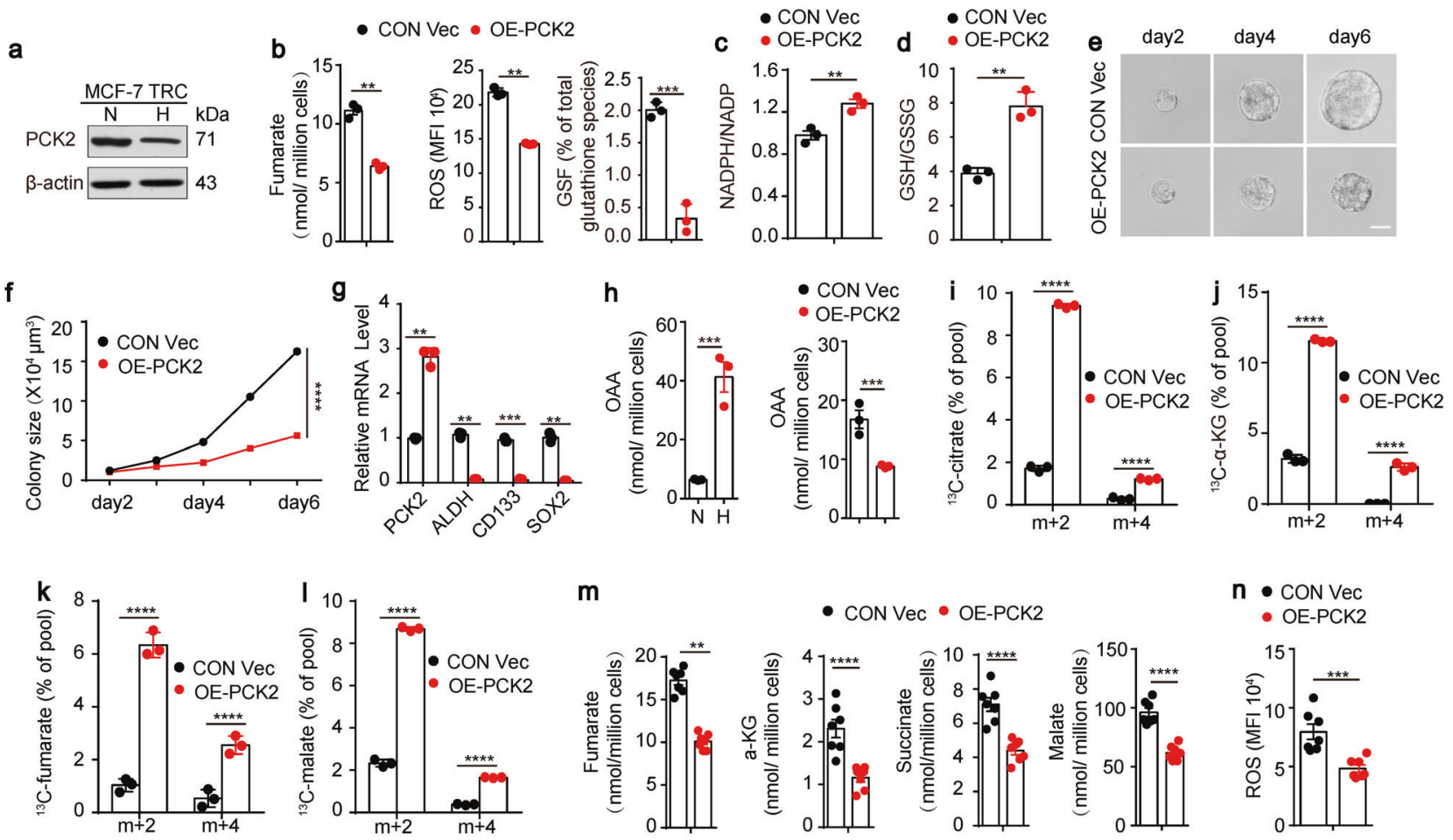

m
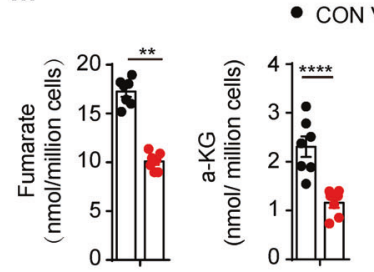

- CON Vec • OE-PCK2

n $\bullet$ CON Vec

- OE-PCK2
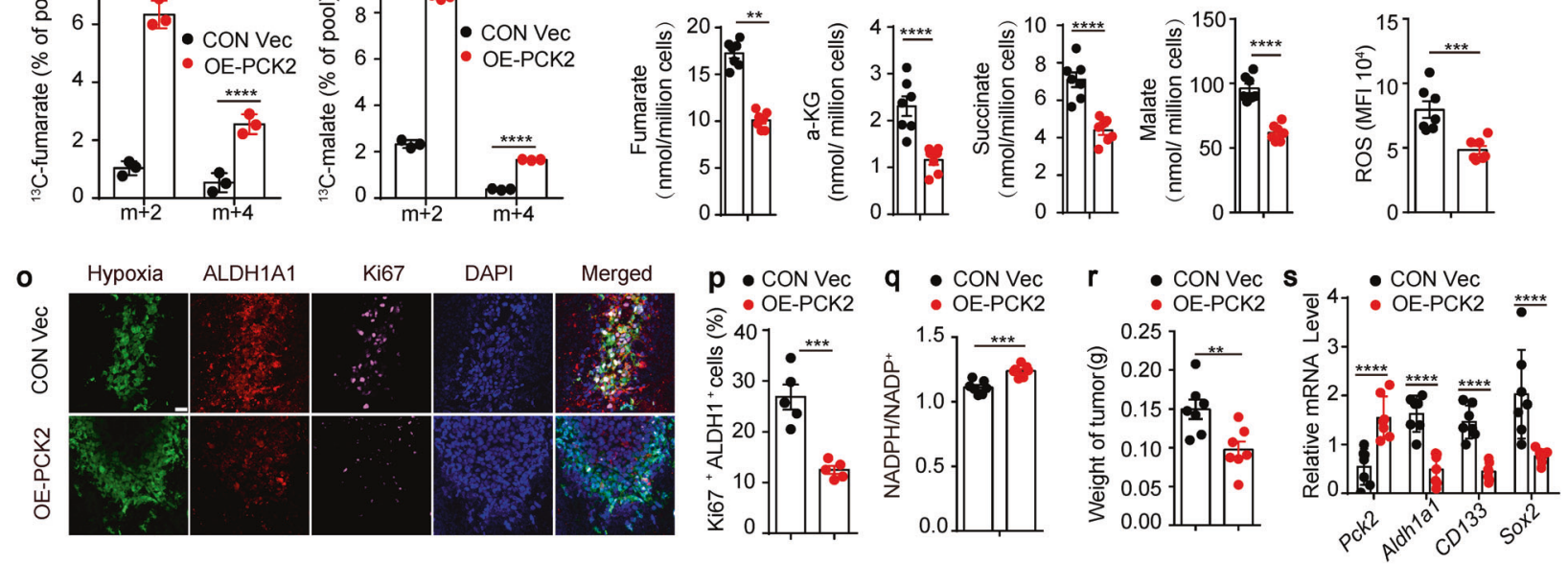

Fig. 6 PCK2 downregulation causes fumarate accumulation. a MCF-7 cells were seeded in $90 \mathrm{~Pa}$ soft 3D fibrin gels for 5 days in $21 \%(\mathrm{~N})$ or $1 \% \mathrm{O}_{2}(\mathrm{H})$ condition and PCK2 expression was determined by western blotting. b Tet-PCK2 MCF-7 cells were cultured in $90 \mathrm{~Pa}$ soft 3D fibrin gels with or without doxycycline in hypoxia for 5 days. Cell extracts were prepared, fumarate and GSF were detected by LC-MS, ROS was detected by flow cytometry. c, d Tet-PCK2 MCF-7 TRCs were treated with or without doxycycline in hypoxia for 5 days. $\mathrm{NADPH} / \mathrm{NADP}^{+}$and GSH/GSSG ratios were analyzed. e, $\mathbf{f}$ Tet-PCK2 MCF-7 cells were cultured in $90 \mathrm{~Pa}$ soft 3D fibrin gels with or without doxycycline in hypoxia for 5 days and the colony size was analyzed. $\mathbf{g}$ Tet-PCK2 MCF-7 cells were cultured in $90 \mathrm{~Pa}$ soft 3D fibrin gels with or without doxycycline in hypoxia for 5 days, Aldh1a1, CD133, and Sox 2 mRNA levels were analyzed by real-time PCR. h OAA levels of normoxic or hypoxic MCF-7 TRCs were analyzed by OAA assay kit as described in methods (left). Tet-PCK2 MCF-7 cells were cultured in $90 \mathrm{~Pa}$ soft 3D fibrin gels with or without doxycycline in hypoxia for 5 days and OAA levels were analyzed (right). i-l Tet-PCK2 MCF-7

regulates heterogeneous tumor cells, however, remains unclear. In particular, how hypoxia reprograms the metabolism of stem-like tumorigenic cells is poorly understood. In the present study, we provide evidence that hypoxia induces the downregulation of PCK2 expression that promotes the growth of human breast tumor-repopulating cells via reprogramming the TCA cycle. cells were seeded in $90 \mathrm{~Pa}$ soft $3 \mathrm{D}$ fibrin gels for 4 days in hypoxia, and then incubated with ${ }^{13} \mathrm{C}$-glucose for another $24 \mathrm{~h}$. $\mathrm{M}+2$ or $\mathrm{m}+4$ labeled citrate, $\alpha-\mathrm{KG}$, fumarate and malate were analyzed by LC-MS. $\mathbf{m}-\mathbf{s}$ Tet-PCK2 MCF-7 TRCs were inoculated into mammary gland of nude mice and mice were treated with sunitinib and doxycycline as described in methods. Thirty-five days after tumor inoculation, ALDH1 + tumor cells were sorted by Aldefluor assay, and then cell extracts were prepared, and TCA cycle intermediates was detected by LC-MS (m), ROS level was detected by flow cytometry (n). Tumor tissue was detected by immunofluorescence staining of pimonidazole adducts, ALDH1A1, Ki67 in sections from control or doxycyclinetreated animals as described in methods $(\mathbf{o}, \mathbf{p})$. The NADPH/NADP + ratios were analyzed as described in methods $(\mathbf{q})$, tumor weight was measured (r) and Aldh1a1, CD133, and Sox2 mRNA levels were analyzed by real-time PCR (s). Data shown are representative of three independent experiments and error bars represent mean \pm SEM. ${ }^{*} p<$ $0.05, * * p<0.01, * * * p<0.001, * * * * p<0.0001$. Scale bars, $100 \mu \mathrm{m}$

Cancer cells show increased ROS in the cytosol, where free radicals promote many aspects of tumor progression and metastasis [40]. Notably, such ROS production can be further elevated under hypoxic conditions. For example, mitochondria increase the levels of cytosolic ROS during hypoxia, where ROS is produced at the Qo site of the mitochondrial complex III [41]. Although many reports 

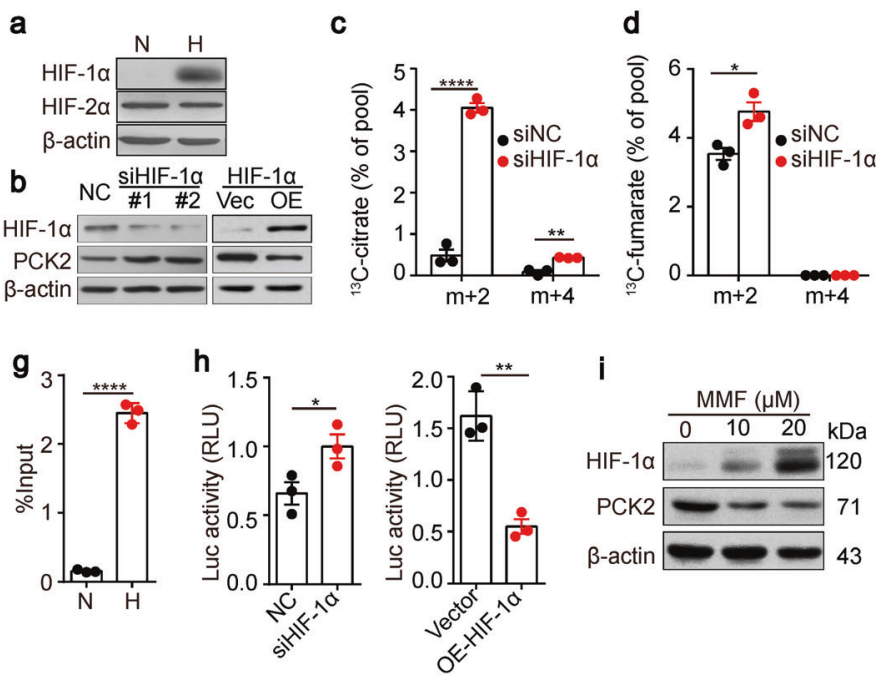

f

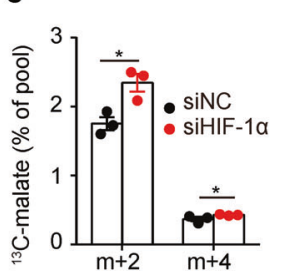

j

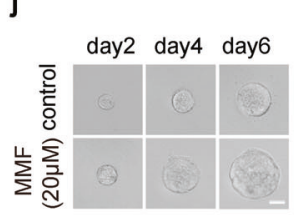

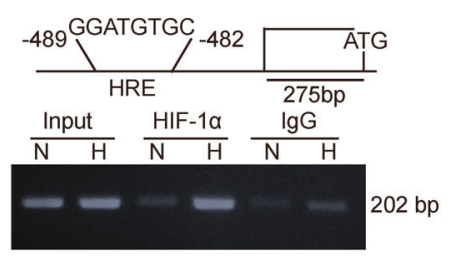

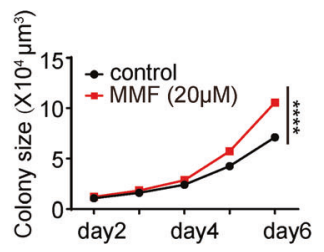

Fig. 7 HIF- $1 \alpha$ negatively regulates PCK2 expression. a HIF- $1 \alpha$ and HIF- $2 \alpha$ expression in normoxic or hypoxic MCF-7 TRCs were analyzed by western blotting. b MCF-7 cells transfected with HIF- $1 \alpha$ siRNA or HIF- $1 \alpha$-overexpressing plasmid were cultured in $90 \mathrm{~Pa}$ soft 3D fibrin gels in hypoxia or normoxia for 5 days, HIF-1 $\alpha$ and PCK2 expression was analyzed by western blotting. c-e MCF-7 cells transfected with HIF- $1 \alpha$ siRNA were seeded in $90 \mathrm{~Pa}$ soft 3D fibrin gels and cultured for 4 days in hypoxic condition, and then incubated with ${ }^{13} \mathrm{C}$-glucose for another $24 \mathrm{~h}$, LC-MS was performed for $\mathrm{m}+2$ or $\mathrm{m}$ +4 labeled citrate, fumarate and malate detection. f, g Schematic representation of the promoter region which located at -489 to $-482 \mathrm{bp}$ on the upstream of the transcription start site of $P c k 2$. MCF-7 TRCs were cultured in $21 \%(\mathrm{~N})$ or $1 \%(\mathrm{H}) \mathrm{O}_{2}$ condition and then HIF- $1 \alpha$

indicate that mitochondrial ROS production is capable of stabilizing HIF- $1 \alpha$ protein during hypoxia [42, 43], studies also show that HIF-1 $\alpha$ stabilization is independent of mitochondrial ROS [42]. Notwithstanding this paradox, to date, the mechanism through which hypoxia increases ROS levels remains elusive [44]. In the present study, we provide evidence that the impairment of the ROS clearance is an important pathway that mediates the elevation of ROS levels under hypoxic condition. Glutathione system plays a critical role in anti-oxidation. Reduced glutathione (GSH) uses the thiol group to react with ROS, concomitant with the conversion of GSH to glutathione disulfide (GSSG). In this process, glutathione reductase is required to catalyze GSSG to GSH with NADPH providing the proton, thus maintaining the continuous clearance of ROS and keeping redox homeostasis. However, hypoxic TRCs attenuate their TCA cycle, leading to fumarate accumulation; the latter then covalently binds to thiolate anion of cysteine residues of glutathione. This succinated GSH acts as an alternative substrate to compete glutathione reductase with GSSG and inhibits GSH regeneration, leading to increasing ROS levels.

High levels of ROS directly oxidize macromolecules, which induces senescence and apoptosis by causing damage to DNA and mitochondrial permeabilization or activating enrichment around the promoter of $P c k 2$ were analyzed by CHIP-PCR as described in methods and $\mathrm{IgG}$ was used as a negative control (f). CHIP-qPCR were used to Pck2 quantitative detection and total genomic DNA was used as input (g). h MCF-7 TRCs were transfected with HIF- $1 \alpha$ siRNA or overexpress HIF- $1 \alpha$ plasmid, and then the luciferase activity was detected as described in methods. i MCF-7 TRCs were treated with different concentrations of DMF $(0,10 \mu \mathrm{M}$, $20 \mu \mathrm{M}$ ) in normoxia, and then HIF-1 $\alpha$ and PCK2 expression were determined by western blotting. j MCF-7 cells were cultured in $90 \mathrm{~Pa}$ soft 3D fibrin gels without or with $20 \mu \mathrm{M}$ DMF in normoxia for five days and the colony size was analyzed. Data shown are representative of three independent experiments and error bars represent mean \pm SEM. $* p<0.05, * * p<0.01, * * * p<0.001, * * * * p<0.0001$

protein kinase $\mathrm{C} \delta$ which triggers senescence [45, 46]. Notwithstanding this ROS-triggered cellular damage, a moderate increase of ROS levels actually exerts a signaling transduction effect. In this study, we find that ROS promotes TRCs growth by activating NF- $\mathrm{kB}$ and Akt pathways, and inhibiting either NF- $\mathrm{KB}$ or Akt blocks the effect of ROS on TRC growth. These findings reveal that breast cancer TRCs mobilize the elevated ROS to activate tumorpromoting signal molecules for their growth during hypoxia. Paradoxically, we also find in this study that hypoxia induces the growth retardation or even apoptosis of differentiated breast cancer cells. These cells have higher ROS than hypoxic TRCs and their growth retardation can be relieved by the addition of NAC, suggesting that excessive ROS has an inhibitory effect on differentiated breast cancer cells. However, the question is why differentiated breast cancer cells produce more ROS during hypoxia than the TRCs. Furthermore, whether and how hypoxia-reprogrammed metabolism differs between differentiated breast cancer cells and TRCs remains unclear. However, these issues are worthy of investigation.

An important finding in this study is that PCK2 triggers hypoxic breast TRC growth. Phosphoenolpyruvate carboxykinase has two isoforms, PCK1 (cytosolic) and PCK2 (mitochondrial). PCK is the hub enzyme that regulates 
glycolysis, tricarboxylic acid cycle and gluconeogenesis. Previously, we found that the upregulation of PCK1 but downregulation of PCK2 mediate the retrograde carbon flow to glycerol-3-phosphate for glycerol biogenesis which fixes free fatty acids to lipids in melanoma TRCs [38, 47]. Such PCK1-directed carbon retrograde flow has also been found in memory $\mathrm{T}$ cells [48]. In this study, we find that PCK2 is downregulated in hypoxic TRCs, thus reducing the transition of mitochondrial OAA to PEP, leading to the accumulation of OAA, and impeding the upstream carbon flow to OAA. In support, the ${ }^{13} \mathrm{C}$ tracing assay shows a hindered carbon flow of the TCA cycle. This might be the reason why we observe an increased fumarate level in hypoxic breast TRCs. However, the question is how PCK2 is downregulated in hypoxic TRCs? Since HIF-1 $\alpha$ is the key transcriptional factor that mediates cellular response to hypoxia, we investigated whether PCK2 was regulated by HIF-1 $\alpha$. Indeed, both Chip-PCR and luciferase analysis showed that HIF- $1 \alpha$ bond to PCK2 promotor region and negatively regulated PCK2 expression. Intriguingly, accumulated fumarate as an $\alpha$-ketoglutarate competing inhibitor, in turn further enhances HIF- $1 \alpha$ stability, leading to further downregulating PCK2 expression and further strengthening fumarate accumulation, thus forming a positive feedback.

In summary, the data in this study clearly show that hypoxia, by virtue of its reprogramming the TCA cycle, promotes the growth of breast TRCs through a fumaratesuccinated GSH-ROS pathway. These findings not only broaden our understanding of the role of hypoxia in cancer biology, but also clarify the differential effect of hypoxia on breast cancer TRCs and the differentiated counterparts. Moreover, this study might possess important clinical significance in that stem-like cancer cells grow more rapidly under the hypoxic conditions. It may explain why cancer recurrence easily occurs following antiangiogenesis therapy. Thus, a combination of an antiangiogenic therapy with an antioxidant modulator is implicated for better tumor treatment.

\section{Materials and methods}

\section{Animals and cell lines}

Female NOD-SCID mice, 6-8 weeks old, were purchased from the Center of Medical Experimental Animals of the Chinese Academy of Medical Sciences (CAMS, Beijing, China). All studies involving mice were approved by the Animal Care and Use Committee of Tongji Medical College. Human MCF-7 and BT474 (breast cancer) cell lines were purchased from the China Center for Type Culture Collection (Beijing, China) and cultured in DMEM (Thermo Scientific) with $10 \%$ fetal bovine serum (FBS) (Gibco, USA). Cells were tested for mycoplasma detection, interspecies cross contamination and authenticated by isoenzyme and short tandem repeat (STR) analyses in the Cell Resource Centre of Peking Union Medical College before the beginning of the study and spontaneously during the research. Before primary tumor tissues collected from patients, informed consent was obtained from all subjects.

\section{Tumor models}

$5 \times 10^{6}$ MCF-7 cells were inoculated into the mammary gland of nude mice. After the tumor grew up to $50 \mathrm{~mm}^{3}$, mice were followed by intragastric administration of $60 \mathrm{mg} /$ $\mathrm{kg}$ sunitinib per day for 35 days. In PCK2 overexpression tumor model, $3 \times 10^{5}$ Tet-PCK2 MCF-7 TRCs were inoculated into the mammary gland of nude mice (control vector transfected MCF-TRCs as control) and which were both treated with $2 \mathrm{mg} / \mathrm{ml}$ doxycycline in drinking water. $90 \mathrm{~min}$ before killing, a pipernoxazole solution $(60 \mathrm{mg} / \mathrm{kg})$ was i.v. injected to label the hypoxic region in vivo.

\section{Immunofluorescence staining}

Tumor tissues were isolated, fixed by $37 \%$ formalin, embedded in paraffin and sectioned for immunofluorescence staining. The sections from paraffin embedded tissues were prepared through dewaxing, antigen retrieval, and acid denaturation before staining. Then sections were incubated with anti-ALDH1A1(1:200, ThermoFisher, USA), anti-pimonidazole (1:50, Hypoxyprobe, USA) and anti-Ki67 (1:50, Biolegend, USA) antibody at $4{ }^{\circ} \mathrm{C}$ overnight. Slides were then incubated sequentially with fluorescence-conjugated secondary antibodies for $1 \mathrm{~h}$ at room temperature. DAPI was stained for nucleus and sections were observed under microscope (Leica TCS SP8 STED).

\section{D rigid dish and 3D fibrin gel cell culture of tumor cells}

For conventional 2D cell culture, tumor cells were maintained in rigid dish with DMEM cell culture medium supplemented with 10\% FBS (Invitrogen, Carlsbad, CA, USA) at $37^{\circ} \mathrm{C}$ with $5 \% \quad \mathrm{CO}_{2}$. TRCs culture were conducted according to our previously described method [27]. In brief, tumor cells were maintained in the conventional rigid plate. After $0.25 \%$ trypsin digestion, cells were detached and suspended in DMEM (10\% FBS) and cell density was adjusted to $10^{4}$ cells $/ \mathrm{ml}$. Fibrinogen (Searun Holdings Company, Freeport, ME, USA) was diluted into $2 \mathrm{mg} / \mathrm{ml}$ with $\mathrm{T}_{7}$ buffer ( $\mathrm{pH} 7.4,50 \mathrm{mM}$ Tris, $150 \mathrm{mM} \mathrm{NaCl}$ ). A 1:1 fibrinogen and cell solution mixture was made by mixing the same volume of the fibrinogen solution and the cell solution, resulting in $1 \mathrm{mg} / \mathrm{ml}$ fibrinogen and 5000 cells $/ \mathrm{ml}$ 
in the mixture. $250 \mu$ cell per fibrinogen mixtures was seeded into each well of a 24 -well plate mixed well with pre-added $5 \mu \mathrm{l}$ thrombin $(0.1 \mathrm{U} / \mathrm{ml}$, Searun Holdings Company). The cell culture plate was then moved into $37^{\circ} \mathrm{C}$ cell culture incubator incubated for $30 \mathrm{~min}$. Finally, $1 \mathrm{ml}$ DMEM medium containing 10\% FBS was added. On day 5, the cells cultured in soft 3D fibrin gels (90 Pascal) were treated with dispase II (Roche, Swiss) for $10 \mathrm{~min}$ at $37^{\circ} \mathrm{C}$ and then the spheroids were collected and pipetted to single cells.

\section{Real-time PCR}

RNAs were isolated with Trizol reagent (Invitrogen) and the cDNAs were generated by reverse transcription (ReverTra Ace qPCR Kit, Toyobo). Real-time PCR was performed for all genes with primers on a Bio-Rad CFX Connet. The expression of mRNA for genes of interest was normalized to $\beta$-actin.

The primer sequences were as follows: hum Aldh1a1, forward 5'-CGGGAAAAGCAATCTGAAGAGGG-3', reverse 5'-GATGCGGCTATACAACACTGGC-3'; Hum Sox2, forward 5'-GCTACAGCATGATGCAGGACCA-3', reverse 5'TCTGCGAGCTGGTCATGGAGTT-3'; hum CD133, forward 5'-CACTACCAAGGACAAGGCGTTC-3', reverse 5'-CAACGCCTCTTTGGTCTCCTTG-3'; hum Oct3/4,5'CCTGAAGCAGAAGAGGATCACC-3', reverse 5'-AAA GCGGCAGATGGTCGTTTGG-3'; hum Klf4, forward 5'CATCTCAAGGCACACCTGCGAA-3', reverse 5'-TCGG TCGCATTTTTGGCACTGG-3'; hum Pck2, forward 5'TAGTGCCTGTGGCAAGACCAAC-3', reverse 5'-GAAG CCGTTCTCAGGGTTGATG-3'; hu-m Mdh2, forward 5'CTGGACATCGTCAGAGCCAACA-3', reverse 5'-GGAT GATGGTCTTCCCAGCATG-3'.

\section{Western blotting}

Cell lysates preparation, SDS-PAGE, electrophoretic transfer, immunoblotting and chemiluminescent detection were performed. Antibodies and dilutions are as follows: PCK2 at 1:1000 (CST, 12940 S), NF-kB p65 (D14E12) at 1:1000 (CST, 8242 S), Phospho-NF-kB p65 (Ser536) (93H1) (CST, $3033 \mathrm{~S})$, Akt (pan)(C67E7) at 1:1000 (CST, $4691 \mathrm{~S}$ ), Phospho-Akt (Ser473) (D9E) at 1:1000 (CST, $4060 \mathrm{~S}$ ), IDH3G at 1:1000 (Proteintech, 25848-1-AP), CS at 1:1000 (CST, 14309 S), OGDH at 1:1000 (Proteintech, 15212-1-AP), HIF-1 $\alpha$ at 1:1000 (Gene Tex, GTX127309), HIF-2 $\alpha$ at 1:1000 (Novus, NB100-132).

\section{RNA interference}

TRCs harvested from fibrin gels by digestion with dispase II $(1 \mathrm{mg} / \mathrm{ml}$, Roche, Mannheim, Germany) were seeded in dishes precoated with fibrin gels for $4 \mathrm{~h}$. Then TRCs were transfected with siRNA via Lipofectamine 2000 (Invitrogen) in Opti-MEM Reduced Serum Medium (Life Technologies, Gaithersburg, MA, USA) as described by the manufacturer at $40 \mathrm{nM}$ final concentration. The siRNAs were synthesized by RiboBio (Guangzhou, China), and the list is shown in Supplementary Table S1.

\section{Chromatin immunoprecipitation}

A chromatin immunoprecipitation (ChIP) assay kit (Active Motif) was utilized to examine the binding of HIF-1 $\alpha$ to the PCK2 promoter. Hypoxic and normoxic MCF-7 TRCs were fixed with $1 \%$ formaldehyde on ice to cross-link the proteins bound to the chromatin DNA. After washing, the chromatin DNA was sheared by enzymatic force to produce DNA fragments of around 200-1000 bp. The same amounts of sheared DNA were used for immunoprecipitation with a HIF-1 $\alpha$ antibody (Gene Tex) or an equal amount of preimmune rabbit $\operatorname{IgG}$ (Gene Tex). The immunoprecipitate then was incubated with protein G Magnetic Beads, and the antibody- protein G Magnetic Beads complex was collected for subsequent reverse cross-linking. The same amount of sheared DNA without antibody precipitation was processed for reverse cross-linking and served as input control. DNA recovered from reverse cross-linking was used for PCR. PCR was performed with primers for the $P c k 2$ promoter (forward, 5'-GCAGGTTGAGACAGGAGAA-3' and reverse, 5'-TGCCATGAAATGGTGGA-3') flanking the $\mathrm{HIF}-1 \alpha$ binding site at $59^{\circ} \mathrm{C}$ for 36 cycles.

\section{${ }^{13} \mathrm{C}$ tracing by liquid chromatography Q-exactive mass spectrometry (LC-QE-MS)}

For ${ }^{13} \mathrm{C}$ tracing experiments, MCF-7 TRCs were cultured in normoxic $\left(21 \% \mathrm{O}_{2}\right)$ and hypoxic $\left(1 \% \mathrm{O}_{2}\right)$ conditions. On day 4 of culture, cells were washed, then cultured with [U6] $-{ }^{13} \mathrm{C}$-glucose for another $24 \mathrm{~h}$. Cells were washed twice in saline and lysed in extraction solvent (80\% methanol/ water) for $30 \mathrm{~min}$ at $-80^{\circ} \mathrm{C}$. After centrifugation at $13,000 \times g, 10 \mathrm{~min}$ at $4{ }^{\circ} \mathrm{C}$, supernatant extracts were analyzed by LC-QE-MS as described previously. In brief, liquid chromatography was performed using a HPLC (Ultimate 3000 UHPLC) system (Thermo Fisher) equipped with An X bridge amide column $(100 \times 2.1 \mathrm{~mm}$ i.d, $3.5 \mu \mathrm{m}$; 97 Waters). The column temperature was maintained at $10^{\circ}$ C. The mobile phase A is $20 \mathrm{mM}$ ammonium acetate and $15 \mathrm{mM}$ ammonium hydroxide in water with $3 \%$ acetonitrile, $\mathrm{pH}$ 9.0, and mobile phase $\mathrm{B}$ is acetonitrile. The linear gradient is as follows: $0 \mathrm{~min}, 85 \% \mathrm{~B} ; 1.5 \mathrm{~min}, 85 \% \mathrm{~B}$, $5.5 \mathrm{~min}, 30 \% \mathrm{~B} ; 8 \mathrm{~min}, 30 \% \mathrm{~B}, 10 \mathrm{~min}, 85 \% \mathrm{~B}$, and $12 \mathrm{~min}$, $85 \%$ B. The flow rate was $0.2 \mathrm{ml} / \mathrm{min}$. Sample volumes of $5 \mu \mathrm{l}$ were injected for LC-MS analysis. LC-MS analysis was 
performed on a Q-exactive mass spectrometer (Thermo Fisher) equipped with a HESI probe, and the relevant parameters are as listed: heater temperature, $120^{\circ} \mathrm{C}$; sheath gas, 30; auxiliary gas, 10; sweep gas, 3; spray voltage, $2.5 \mathrm{kV}$ for the negative mode. A full scan ranges from 80 to $350(\mathrm{~m} / \mathrm{z})$ was used. The resolution was set at 70,000. Data were quantified by integrating the area underneath the curve of each compound using X calibur Qual browser (Thermo Fisher). Each metabolite's accurate mass ion and subsequent isotopic ions were extracted (EIC) using a 10-ppm window.

\section{LC-MS conditions to detect succinated GSH}

The experiment was performed using a HPLC (Ultimate 3000 UHPLC) system (Thermo Fisher) equipped with An X bridge amide column $(100 \times 2.1 \mathrm{~mm}$ i.d, $3.5 \mu \mathrm{m} ; 97$ Waters). The column temperature was maintained at $10{ }^{\circ} \mathrm{C}$. The mobile phase $\mathrm{A}$ is $20 \mathrm{mM}$ ammonium acetate and $15 \mathrm{mM}$ ammonium hydroxide in water with $3 \%$ acetonitrile, $\mathrm{pH}$ 9.0, and mobile phase $\mathrm{B}$ is acetonitrile. The linear gradient is as follows: $0 \mathrm{~min}, 90 \% \mathrm{~B} ; 2 \mathrm{~min}, 70 \% \mathrm{~B}, 4 \mathrm{~min}$, $50 \% \mathrm{~B} ; 7 \mathrm{~min}, 50 \% \mathrm{~B}$, and $9 \mathrm{~min}, 10 \% \mathrm{~B}$. The flow rate was $0.2 \mathrm{ml} / \mathrm{min}$. Sample volumes of $5 \mu \mathrm{l}$ were injected for LCMS analysis. LC-MS analysis was performed on a Qexactive mass spectrometer (Thermo Fisher) equipped with a HESI probe, and the relevant parameters are as listed: heater temperature, $120^{\circ} \mathrm{C}$; sheath gas, 30 ; auxiliary gas, 10 ; sweep gas, 3 ; spray voltage, $2.5 \mathrm{kV}$ for the negative mode. A full scan ranges from 80 to $350(\mathrm{~m} / \mathrm{z})$ was used. The resolution was set at 70,000 . Data were quantified by integrating the area underneath the curve of each compound using X calibur Qual browser (Thermo Fisher). Each metabolite's accurate mass ion and subsequent isotopic ions were extracted (EIC) using a 10-ppm window. The mass spectrometry (MS) was operated in the negative mode and multiple reactions monitoring (MRM). All dependent MS parameters were optimized, based on infusion experiments. The tandem transition from the parent ions of $[\mathrm{M}-\mathrm{H}]-$ to the fragment ions of $71 \mathrm{~m} / \mathrm{z}$ for fumaric acid (FUM), $272 \mathrm{~m} /$ $\mathrm{z}$ for glutathione reduced (GSH) and oxidized (GSSG), were monitored for quantitative analysis. Data were acquired and analyzed using Analyst software version 1.6. Methods to quantify succinated GSH are provided in supplemental procedures.

\section{Oxaloacetate assay}

For the analysis of the concentration of OAA, MCF-7 TRCs or control cells $\left(1 \times 10^{6}\right)$ were washed with ice-cold phosphate-buffered saline and were then rapidly homogenized in $100 \mu \mathrm{l}$ of OAA assay buffer. Samples were centrifuged at $15,000 \times g$ for $10 \mathrm{~min}$ to remove insoluble materials.
Supernatants were assayed with the OAA Assay Kit (MAK070, Sigma) according to the manufacturer's instructions.

\section{ROS detection}

ROS levels were measured using CellROX ${ }^{\circledR}$ Green flow cytometry assay kits (Life technologies). Cells were loaded with $500 \mathrm{nM}$ CellROX ${ }^{\circledast}$ Green for $30 \mathrm{~min}$ at $37^{\circ} \mathrm{C}$, protected from light. Cells were washed and scraped in PBS and immediately analyzed by flow cytometry, using $488 \mathrm{~nm}$ excitation for the CellROX ${ }^{\circledR}$ Green.

\section{NADPH/NADP + and GSH/GSSG assay}

The NADPH/NADP ${ }^{+}$and GSH/GSSG ratio were determined with the NADP/NADPH Quantification Colorimetric Kit and Glutathione Fluorometric Assay Kit respectively (both form BioVision). Measurements were performed according to the manufacturer's instructions.

\section{Aldefluor assay and flow cytometry}

The Aldefluor assay was carried out according to the manufacturer's (STEMCELL Technologies) guidelines. Briefly, cells were suspended in Aldefluor assay buffer containing an ALDH substrate, bodipy-amino acetaldehyde, at $1.5 \mu \mathrm{M}$, and incubated for $45 \mathrm{~min}$ at $37^{\circ} \mathrm{C}$. To distinguish between $\mathrm{ALDH}^{+}$and $\mathrm{ALDH}^{-}$cells, a fraction of cells was incubated with a 10-fold excess of an ALDH inhibitor, diethylamino- benzaldehyde. This results in a significant decrease in fluorescence intensity of $\mathrm{ALDH}^{+}$cells and were used to compensate the flow cytometer.

\section{Luciferase assays}

MCF-7 cell lines, stably integrated with a pGV238-PCK2 promoter-driven luciferase, were treated in hypoxic and normoxic for $48 \mathrm{~h}$. Then, these cells were lysed and analyzed by a luciferase assay using the luciferase assay kit (Promega, WI, USA) on a GloMax Multi Plus (Promega). MCF-7 cells were transfected with 100 ng Renilla luciferase, $1 \mu \mathrm{g}$ firefly luciferase plasmid pGV238-PCK2 promoter-luc and $1 \mu \mathrm{g}$ of either vector or pCMV6-HIF-1 $\alpha$ for $12 \mathrm{~h}$. Then, these cells were cultured for $36 \mathrm{~h}$. Cells lysates were analyzed using the Dual Luciferase Reporter Assay (Promega) on a GloMax Multi Plus (Promega). Firefly luciferase activity was normalized to Renilla luciferase.

\section{Cell cycle analysis}

Cells were incubated with $20 \mathrm{mM}$ BrdU (BD Bioscience) for $1 \mathrm{~h}$ and cell cycle analysis was performed using BD 
Pharmingen FITC-BrdU Flow Kits according to the manufacturer's protocol (BD Bioscience, NJ, USA). The samples were analyzed by flow cytometry on a BD Accuri C6 Flow Cytometer (BD Bioscience). The following cell cycle phases were determined as a percentage of the total population: sub-G0 (apoptotic cells), G0/G1 (2n, BrdU-negative), $S$ ( $2 n$ to $4 n$, BrdU-positive) and $G_{2} / M$ phase ( $4 n$, BrdU-negative). The primary tumor cells were isolated from tumor or ascites and used for cell cycle analysis.

\section{Statistics analysis}

All experiments were performed at least three times and the mice were randomly divided into groups in all animal studies. To determine the colony size, at least 20 colonies from different field were measured. Results are expressed as mean \pm SEM. and analyzed by two-sided Student's $t$-test. The $P$ value $<0.05$ was considered statistically significant. The analysis was conducted using the GraphPad 6.0 software. Sample exclusion was never carried out. The investigator was blinded to the group allocation during the experiment and when assessing the outcome.

\section{Data availability}

The authors declare that all the data supporting the findings of this study are available within the article and its Supplementary Information files and from the corresponding author on reasonable request.

Acknowledgements This work was supported by National Natural Science Foundation of China (81788101, 81530080, and 81601366), CAMS Initiative for Innovative Medicine (2016-I2M-1-007).

Author contributions B.H. conceived the project. K.T., Y.Y., L.Z., J. C., P.X., H.Z., J.M., K.W., H.F., F.L., J.Z., J.X., J.J. and Y.L. performed the experiments. B.H., Y.Y., K.T., H.Z., J.M. and L.Z developed methodology. B.H., Y.Y, K.T., W.S., J.C. and L.Z. performed data analysis. K.T. provided administrative, technical, or material support. B.H., K.T. and Y.Y. wrote the manuscript with input from all authors.

\section{Compliance with ethical standards}

Conflict of interest The authors declare that they have no conflict of interest.

Publisher's note: Springer Nature remains neutral with regard to jurisdictional claims in published maps and institutional affiliations.

Open Access This article is licensed under a Creative Commons Attribution 4.0 International License, which permits use, sharing, adaptation, distribution and reproduction in any medium or format, as long as you give appropriate credit to the original author(s) and the source, provide a link to the Creative Commons license, and indicate if changes were made. The images or other third party material in this article are included in the article's Creative Commons license, unless indicated otherwise in a credit line to the material. If material is not included in the article's Creative Commons license and your intended use is not permitted by statutory regulation or exceeds the permitted use, you will need to obtain permission directly from the copyright holder. To view a copy of this license, visit http://creativecommons. org/licenses/by/4.0/.

\section{References}

1. Wilson WR, Hay MP. Targeting hypoxia in cancer therapy. Nat Rev Cancer. 2011;11:393-410.

2. Bertout JA, Patel SA, Simon MC. The impact of $\mathrm{O}_{2}$ availability on human cancer. Nat Rev Cancer. 2008;8:967-75.

3. Hou HG, Khan N, Du GX, Hodge S, Swartz HM. Temporal variation in the response of tumors to hyperoxia with breathing carbogen and oxygen. Med Gas Res. 2016;6:138-46.

4. Ostergaard L, Tietze A, Nielsen T, Drasbek KR, Mouridsen K, Jespersen $\mathrm{SN}$, et al. The relationship between tumor blood flow, angiogenesis, tumor hypoxia, and aerobic glycolysis. Cancer Res. 2013;73:5618-24.

5. Ghajar CM. Metastasis prevention by targeting the dormant niche. Nat Rev Cancer 2015;15:238-47.

6. McIntyre A, Patiar S, Wigfield S, Li JL, Ledaki I, Turley H, et al. Carbonic anhydrase IX promotes tumor growth and necrosis in vivo and inhibition enhances anti-VEGF therapy. Clin Cancer Res. 2012;18:3100-11.

7. Olcina MM, Leszczynska KB, Senra JM, Isa NF, Harada $H$, Hammond EM. H3K9me3 facilitates hypoxia-induced p53dependent apoptosis through repression of APAK. Oncogene. 2016;35:793-9.

8. Gilkes DM, Semenza GL, Wirtz D. Hypoxia and the extracellular matrix: drivers of tumour metastasis. Nat Rev Cancer. 2014;14:430-9.

9. Muz B, de la Puente P, Azab F, Azab AK. The role of hypoxia in cancer progression, angiogenesis, metastasis, and resistance to therapy. Hypoxia. 2015;3:83-92.

10. Bruna A, Rueda OM, Greenwood W, Batra AS, Callari M, Batra $\mathrm{RN}$, et al. A biobank of breast cancer explants with preserved intra-tumor heterogeneity to screen anticancer compounds. Cell. 2016;167:260-74

11. Koren S, Bentires-Alj M. Breast tumor heterogeneity: source of fitness, hurdle for therapy. Mol Cell. 2015;60:537-46.

12. Lou Y, McDonald PC, Oloumi A, Chia S, Ostlund C, Ahmadi A, et al. Targeting tumor hypoxia: suppression of breast tumor growth and metastasis by novel carbonic anhydrase IX inhibitors. Cancer Res. 2011;71:3364-76.

13. Montagner M, Enzo E, Forcato M, Zanconato F, Parenti A, Rampazzo E, et al. SHARP1 suppresses breast cancer metastasis by promoting degradation of hypoxia-inducible factors. Nature. 2012;487:380-4.

14. Wong CC, Gilkes DM, Zhang H, Chen J, Wei H, Chaturvedi P, et al. Hypoxia-inducible factor 1 is a master regulator of breast cancer metastatic niche formation. Proc Natl Acad Sci USA. 2011;108:16369-74.

15. Man J, Yu X, Huang H, Zhou W, Xiang C, Huang H, et al. Hypoxic induction of vasorin regulates notch1 turnover to maintain glioma stem-like cells. Cell Stem Cell. 2018;22:104-18.

16. Xing F, Okuda H, Watabe M, Kobayashi A, Pai SK, Liu W, et al. Hypoxia-induced Jagged2 promotes breast cancer metastasis and self-renewal of cancer stem-like cells. Oncogene. 2011;30:4075-86.

17. Brooks MD, Burness ML, Wicha MS. Therapeutic implications of cellular heterogeneity and plasticity in breast cancer. Cell Stem Cell. 2015;17:260-71.

18. Conley SJ, Gheordunescu E, Kakarala P, Newman B, Korkaya H, Heath AN, et al. Antiangiogenic agents increase breast cancer 
stem cells via the generation of tumor hypoxia. Proc Natl Acad Sci USA. 2012;109:2784-9.

19. Samanta D, Park Y, Andrabi SA, Shelton LM, Gilkes DM, Semenza GL. PHGDH expression is required for mitochondrial redox homeostasis, breast cancer stem cell maintenance, and lung metastasis. Cancer Res. 2016;76:4430-42.

20. Semenza GL. Hypoxia-inducible factors: coupling glucose metabolism and redox regulation with induction of the breast cancer stem cell phenotype. EMBO J. 2017;36:252-9.

21. Hitosugi T, Fan J, Chung TW, Lythgoe K, Wang X, Xie J, et al. Tyrosine phosphorylation of mitochondrial pyruvate dehydrogenase kinase 1 is important for cancer metabolism. Mol Cell. 2011;44:864-77.

22. Al-Hajj M, Wicha MS, Benito-Hernandez A, Morrison SJ, Clarke MF. Prospective identification of tumorigenic breast cancer cells. Proc Natl Acad Sci USA. 2003;100:3983-8.

23. Clevers $H$. The cancer stem cell: premises, promises and challenges. Nat Med. 2011;17:313-9.

24. Magee JA, Piskounova E, Morrison SJ. Cancer stem cells: impact, heterogeneity, and uncertainty. Cancer Cell. 2012;21:283-96.

25. Medema JP. Cancer stem cells: the challenges ahead. Nat Cell Biol. 2013;15:338-44.

26. Chaffer CL, Brueckmann I, Scheel C, Kaestli AJ, Wiggins PA, Rodrigues LO, et al. Normal and neoplastic nonstem cells can spontaneously convert to a stem-like state. Proc Natl Acad Sci USA. 2011;108:7950-5.

27. Liu J, Tan Y, Zhang H, Zhang Y, Xu P, Chen J, et al. Soft fibrin gels promote selection and growth of tumorigenic cells. Nat Mater. 2012;11:734-41.

28. Dieter SM, Ball CR, Hoffmann CM, Nowrouzi A, Herbst F, Zavidij $\mathrm{O}$, et al. Distinct types of tumor-initiating cells form human colon cancer tumors and metastases. Cell Stem Cell. 2011;9:357-65.

29. Liu Y, Lv J, Liu J, Liang X, Jin X, Xie J, et al. STAT3/p53 pathway activation disrupts IFN-beta-induced dormancy in tumorrepopulating cells. J Clin Invest. 2018;128:1057-73.

30. Liu Y, Liang X, Dong W, Fang Y, Lv J, Zhang T, et al. Tumorrepopulating cells induce PD-1 expression in CD8(+) T cells by transferring kynurenine and AhR activation. Cancer Cell. 2018;33:480-94.

31. Shadel GS, Horvath TL. Mitochondrial ROS signaling in organismal homeostasis. Cell. 2015;163:560-9.

32. Lee CU, Hahne G, Hanske J, Bange T, Bier D, Rademacher C, et al. Redox modulation of PTEN phosphatase activity by hydrogen peroxide and bisperoxidovanadium complexes. Angew Chem Int Ed Engl. 2015;54:13796-800.

33. Papandreou I, Cairns RA, Fontana L, Lim AL, Denko NC. HIF-1 mediates adaptation to hypoxia by actively downregulating mitochondrial oxygen consumption. Cell Metab. 2006;3:187-97.

34. Yang C, Ko B, Hensley CT, Jiang L, Wasti AT, Kim J, et al. Glutamine oxidation maintains the TCA cycle and cell survival during impaired mitochondrial pyruvate transport. Mol Cell. 2014;56:414-24.

35. Zheng L, Cardaci S, Jerby L, MacKenzie ED, Sciacovelli M, Johnson TI, et al. Fumarate induces redox-dependent senescence by modifying glutathione metabolism. Nat Commun. 2015;6:6001.

36. Alderson NL, Wang Y, Blatnik M, Frizzell N, Walla MD, Lyons TJ, et al. S-(2-Succinyl) cysteine: a novel chemical modification of tissue proteins by a Krebs cycle intermediate. Arch Biochem Biophys. 2006;450:1-8.

37. Sullivan LB, Martinez-Garcia E, Nguyen H, Mullen AR, Dufour E, Sudarshan S, et al. The proto-oncometabolite fumarate binds glutathione to amplify ROS-dependent signaling. Mol Cell. 2013;51:236-48.

38. Luo S, Li Y, Ma R, Liu J, Xu P, Zhang H, et al. Downregulation of PCK2 remodels tricarboxylic acid cycle in tumor-repopulating cells of melanoma. Oncogene. 2017;36:3609-17.

39. Vincent EE, Sergushichev A, Griss T, Gingras MC, Samborska B, Ntimbane T, et al. Mitochondrial phosphoenolpyruvate carboxykinase regulates metabolic adaptation and enables glucoseindependent tumor growth. Mol Cell. 2015;60:195-207.

40. Liou GY, Storz P. Reactive oxygen species in cancer. Free Radic Res. 2010;44:479-96.

41. Bell EL, Emerling BM, Ricoult SJ, Guarente L. SirT3 suppresses hypoxia inducible factor 1alpha and tumor growth by inhibiting mitochondrial ROS production. Oncogene. 2011;30:2986-96.

42. Chua YL, Dufour E, Dassa EP, Rustin P, Jacobs HT, Taylor CT, et al. Stabilization of hypoxia-inducible factor-1alpha protein in hypoxia occurs independently of mitochondrial reactive oxygen species production. J Biol Chem. 2010;285:31277-84.

43. Sullivan LB, Chandel NS. Mitochondrial reactive oxygen species and cancer. Cancer Metab. 2014;2:17.

44. Sabharwal SS, Schumacker PT. Mitochondrial ROS in cancer: initiators, amplifiers or an Achilles' heel? Nat Rev Cancer. 2014;14:709-21.

45. Garcia-Perez C, Roy SS, Naghdi S, Lin X, Davies E, Hajnóczky G. Bid-induced mitochondrial membrane permeabilization waves propagated by local reactive oxygen species (ROS) signaling. Proc Natl Acad Sci USA. 2012;109:4497-502.

46. Weyemi U, Lagente-Chevallier O, Boufraqech M, Prenois F, Courtin F, Caillou B, et al. ROS-generating NADPH oxidase NOX4 is a critical mediator in oncogenic H-Ras-induced DNA damage and subsequent senescence. Oncogene. 2012;31:1117-29.

47. Li Y, Luo S, Ma R, Liu J, Xu P, Zhang H, et al. Upregulation of cytosolic phosphoenolpyruvate carboxykinase is a critical metabolic event in melanoma cells that repopulate tumors. Cancer Res. 2015;75:1191-6.

48. Ma R, Ji T, Zhang H, Dong W, Chen X, Xu P, et al. A Pck1directed glycogen metabolic program regulates formation and maintenance of memory $\mathrm{CD}^{+} \mathrm{T}$ cells. Nat Cell Biol. 2018;20:21-7. 